\title{
Sensory processing dysregulations as reliable translational biomarkers in SYNGAPI haploinsufficiency
}

Maria Isabel Carreño-Muñoz ${ }^{1,2}$, Bidisha Chattopadhyaya ${ }^{1}$, Kristian Agbogba ${ }^{1}$, Valérie Côté ${ }^{1,3}$, Siyan Wang ${ }^{4}$, Maxime Lévesque ${ }^{4}$, Massimo Avoli, Jacques L. Michaud ${ }^{1,2,5}$, Sarah Lippé 1,3,*, Graziella Di Cristo ${ }^{1,2, *}$

\section{Affiliations:}

${ }^{1}$ Centre de Recherche, CHU Sainte-Justine (CHUSJ)

${ }^{2}$ Department of Neurosciences, Université de Montréal

${ }^{3}$ Department of Psychology, Université de Montréal

${ }^{4}$ Departments of Neurology and Neurosurgery, Montreal Neurological Institute-Hospital, McGill University

${ }^{5}$ Department of Pediatrics, Université de Montréal

*Corresponding authors:

Graziella Di Cristo,

Centre de Recherche, CHU Sainte-Justine,

3175, Côte-Sainte-Catherine, Montréal, QC H3T 1C5, Canada.

E-mail: graziella.di.cristo@umontreal.ca

Sarah Lippé

Centre de Recherche, CHU Sainte-Justine, 3175,

Côte-Sainte-Catherine, Montréal, QC H3T 1C5, Canada.

E-mail: sarah.lippe@umontreal.ca 


\begin{abstract}
Amongst the numerous genes associated with intellectual disability, SYNGAP1 stands out for its frequency and penetrance of loss-of-function variants found in patients, as well as the wide range of co-morbid disorders associated with its mutation. Most studies exploring the pathophysiological alterations caused by Syngapl haploinsufficiency in mouse models have focused on cognitive problems and epilepsy, however whether and to what extent sensory perception and processing are altered by Syngap1 haploinsufficiency is less clear. By performing EEG recordings in awake mice, we identified specific alterations in multiple aspects of auditory and visual processing, including increased baseline gamma oscillation power, increased theta/gamma phase amplitude coupling following stimulus presentation and abnormal neural entrainment in response to different sensory modality-specific frequencies. We also report lack of habituation to repetitive auditory stimuli and abnormal deviant sound detection. Interestingly, we found that most of these alterations are present in human patients as well, thus making them strong candidates as translational biomarkers of sensory-processing alterations associated with SYNGAP1/Syngapl haploinsufficiency.
\end{abstract}




\section{Introduction}

SYNGAP1 haploinsufficiency-related intellectual disability (SYNGAP1-ID) is characterized by moderate to severe developmental delay or intellectual disability (ID), generalized epilepsy (>80\%), autism spectrum disorder (ASD) and other behavioral abnormalities including severely delayed or absent language development, stereotypic behaviors, poor social development, impulsivity, inattention, aggressive behavior, elevated pain threshold and sleep disorders $(>50 \%)^{1-}$

8. To date more than 700 individuals with SYNGAP1-ID have been reported worldwide through genetic sequencing and this number is rising rapidly due to more widespread genetic testing. The majority of affected individuals carry de novo nonsense or frameshift variants that likely result in haploinsufficiency. SYNGAP1 haploinsufficiency may explain up to $1 \%$ of non-syndromic ID cases $^{2,3,9,10}$, currently making it one of the most frequent causes of genetically-defined childhood brain disorders.

Using rodent models both in vitro and in vivo, research over the last twenty years has uncovered the critical roles played by SYNGAP1, the Ras GTPase-activating protein coded by the Syngapl gene, in neuronal physiology, brain development, and cognition (recently reviewed by Gamache and collaborators ${ }^{11}$ ). Critical to our understanding of SYNGAP1 function in the context of neurodevelopmental disorders, it has been proposed that Syngapl heterozygous knockout mice, which have $\sim 50 \%$ reduction in SYNGAP1 protein levels ${ }^{12}$, offer both construct and face validity for SYNGAP1-ID ${ }^{13}$. Several studies have attempted, and succeeded, to rescue specific behavioral and neurological phenotypes in these mice ${ }^{14,15}$. These exciting results deepen our neurobiological understanding of SYNGAP1 function in normal and haploinsufficient conditions. Nevertheless, a critical, and still missing, step for the successful development of therapies for SYNGAPI-ID is the identification of robust, replicable and translatable biomarkers in humans and animal models.

Electroencephalography (EEG) recordings and sensory-evoked event-related potentials are particularly well suited for the investigation of neurodevelopmental disorders ${ }^{16}$. Since EEG has a high temporal resolution, sensory-evoked related potentials can provide detailed brain-related readouts of cognitive processes. EEG is also non-invasive, portable, inexpensive, and can assess brain activity through passive tasks, and it thereby not dependent on patient's active participation. 
Sensory-evoked potentials exhibit distinctive temporal patterns of activity in specific brain regions that are replicable across trials and between typical individuals ${ }^{16}$. The reliability of EEG as an outcome measure in clinical trials is currently under investigation. Here, we assess whether EEG can be used as a translational (human-mice) investigation tool in SYNGAP1-ID.

Sensory-evoked potentials induced by a discrete sensory stimulus, usually visual or auditory, are currently being investigated as biomarkers of sensory processing impairment in neurodevelopmental disorders ${ }^{16-20}$. In fact, recent studies suggest that sensory-processing impairments are very frequent in neurodevelopmental disorders; they significantly affect social adaptation and behavioural preferences, and limit quality of life ${ }^{19,21}$. A recent study reported abnormal tactile processing in individuals with SYNGAPI-ID, and severe impairments in somatosensory cortex physiology in Syngapl haploinsufficient mice ${ }^{22}$. Several parallel studies on the neurobiological alterations caused by SYNGAP1/Syngapl haploinsufficiency has focused on epileptic activity and cognitive problems. However, whether sensory processing, in particular auditory and visual processing, is impaired in both SYNGAP1-ID individuals and Syngapl haploinsufficient mice is as yet unexlored.

Here, we performed and analyzed EEG recording following different visual and auditory stimulation protocols in awake Syngap1 ${ }^{+/-}$(haploinsufficient) mice and control littermates of both sexes. We further performed the same analysis in a cohort of patients with SYNGAPI-ID and of neurotypic age-matched individuals, who underwent EEG recording following both visual and auditory stimulations. We identified significantly increased gamma oscillation power at baseline consistent across different cortical regions, lack of habituation to repetitive auditory stimuli and increased coupling of theta and gamma oscillations following both visual and auditory stimulations as robust and translational sensory-processing alterations associated with SYNGAP1/Syngapl haploinsufficiency.

\section{Material and Methods}

\section{EEG recordings in mice}

Animals. Experimental procedures involving mice were approved by the Comité Institutionnel de Bonnes Pratiques Animales en Recherche (CIBPAR) of the Research Center of Sainte-Justine Hospital in accordance with the principles published by the Canadian Council on Animal Care. Syngap1 ${ }^{+/-}$mice were kindly provided by Dr Seth Grant (Edinburgh University, United Kingdom) ${ }^{23}$. These carry a deletion of 
exons 8-10 of the Syngap1 gene, which encode for the C2 and GAP domains ${ }^{23}$. They were maintained as a heterozygous line onto a C57B1/6 background. Twenty-two adult Syngap1 ${ }^{+/-}$mice (12 females and 10 males) were used in this study. Mice were implanted at postnatal day (P)70-90 and recorded between P80 and P120. All animals were housed with 2-5 mice/cage from weaning until surgery, after which they were housed singularly for the duration of the experiment. Animals were kept on a $12 \mathrm{~h} / 12 \mathrm{~h}$ light/dark cycle, provided with nesting material and food and water ad libitum. All experiments were performed during the light period under constant mild luminosity (60 Lux). We recorded mice of both sexes. We checked and noted the exact stage of females' estrous cycle after each recording session, by histological inspection of vaginal smear ${ }^{24}$. Here, we analyzed EEG only for females in the pro-estrus/estrus phase, with the exception of inter-ictal spike analysis for which we pulled together data from mice in either diestrus/metestrus or proestrus/estrus recordings.

Surgery. Cranial surgery was performed under deep anesthesia, induced by an intraperitoneal injection of ketamine/xylazine cocktail. A craniotomy was performed at the coordinates described below on the right side of the brains and custom-made clusters of four to six $50 \mu \mathrm{m}$-diameterinsulated-tungsten wires were implanted. In each cluster, wires tips were 50 to $150 \mu \mathrm{m}$ apart. A custom-designed and 3D printed microdrive-like scaffold held the 3 electrode clusters at fixed positions (Supplementary Figure 1), thus allowing the simultaneous implants of all 3 and reducing surgery time.

Data was acquired with a custom-made headstage using Intan Technologies' RHD Electrophysiology Amplifier chip. A ground-and-reference wire was also gently introduced in the contralateral frontal lobe and a $2 \mathrm{~cm}$ carbon fiber bar was placed on the back of the skull. The microdrive apparatus, the carbon fiber bar and the ground-and-reference wire were then daubed with dental acrylic to encase the electrode-microdrive assembly and anchor it to the skull. For one week after surgery, animals were treated with Metacam and the skin around the implant was disinfected daily.

Electrode clusters were implanted at the following coordinates: Primary Auditory Cortex (A1), $2.5 \mathrm{~mm}$ posterior to Bregma, $3.9 \mathrm{~mm}$ lateral to the midline and $0.8 \mathrm{~mm}$ deep; Primary Visual Cortex (V1), -4 mm posterior to Bregma and $2 \mathrm{~mm}$ lateral to the midline; Posterior Parietal Cortex (PPC), -2 mm posterior to Bregma and $1.7 \mathrm{~mm}$ lateral to the midline. Clusters placed in V1 and PPC targeted both supra and infragranular layers, those placed in A1 targeted layer 5. PPC cluster also included 2 longer (1.2 and $1.3 \mathrm{~mm}$ ) wires reaching the dorsal region of the hippocampus (CA1 pyramidal layer and radiatum). Correct depth in CA1 was confirmed by the presence of ripples, 
which appeared on the recordings when the electrode reached the pyramidal layer. For this study, we analyzed only signals from cortical layer 5 and hippocampal radiatum layer.

EEG recording. EEG recordings were performed using an open-ephys GUI platform (https://openephys.org/). Custom designed auditory and visual trigger generator devices sent triggers directly to the recording system assuring the time precision of the stimuli presentation. Starting one week after surgery, animals were habituated daily for 4 days to an open field (15-20' each time), before being recorded during auditory stimulation protocols from day 5 . We used a dimly illuminated open field environment $(45 \times 35 \mathrm{~cm})$, surrounded by $60 \mathrm{~cm}$-high transparent walls, and equipped with video monitoring. The walls were sprayed and wiped clean with $70 \%$ ethanol 30 minutes before the introduction of each animal. Two loudspeakers were placed $\sim 10 \mathrm{~cm}$ from the walls of the open field. Baseline activity was recorded at the beginning of each session for a period of 10 minutes while mice were freely exploring the open field. EEG recoding during auditory stimulations were conducted for 20-30 minutes, a total of 4 times, on alternate days. Mice were then habituated for 7-10 days to the head-fixed system situated on air-supported ball treadmill. This custom-made apparatus allowed the animal to run using a larger range of directions than a classical treadmill. Habituation period was progressively longer, starting from 5 up to 30' daily. Once mice were habituated to stay on the ball for 30 minutes, they were recorded during visual stimulation protocols. Each recording session lasted from 15-30 minutes.

\section{Auditory stimulations protocols}

All stimulations were presented at an intensity of $70 \mathrm{~dB}$. For pure tone stimulation, we used 100 ms long $5 \mathrm{KHz}$ and $10 \mathrm{KHz}$ pure tones presented with a random inter-stimuli interval of 2 to $3 \mathrm{~s}$. Each pure tone was presented 60 times using a random order. The full protocol lasted 5 minutes. For the habituation/oddball paradigm, we used sequences of 5-9 repetitive (standard, at 5KHz) sounds followed by a deviant sound (at $10 \mathrm{KHz}$ ). Sequences were presented in a pre-established pseudorandom order. Sounds lasted $70 \mathrm{~ms}$ and were presented with a $1 \mathrm{~s}$ inter-stimulus-interval. The full protocol lasted 10 minutes. For Auditory Steady State stimulation, we used 10 and $40 \mathrm{~Hz}$ click trains (1s duration and 2.5 inter trial interval) presented with alternating frequencies. Each click was a sound at $5 \mathrm{KHz}$ lasting $5 \mathrm{~ms}$. This protocol lasted for 5 minutes. Finally, we used a Chirp stimulation, adapted from ${ }^{25}$. Briefly, we used a $2 \mathrm{~s} 5 \mathrm{KHz}$ tone whose amplitude was modulated by a sinusoid whose frequency increases (up chirp) or decrease (down chirp) linearly in the 1-100Hz. To avoid onset contaminating phase locking to the amplitude modulation of the chirp, the stimulus 
was ramped in sound level from $0-100 \%$ of its original intensity over 1 s, which then transitioned into chirp modulation sequence mentioned previously. Inter-trial intervals were random in a range from 2-4s. Up and down chirp were presented 50 times each, in alternation. Both directions of modulation were tested to ensure any frequency specific effects were not due to the frequency transition history within the stimulus. The interval between each train was randomly varied between 1 and $1.5 \mathrm{~s}$. The total duration of this protocol was 15 minutes.

\section{Visual stimulation protocols}

All visual stimulations were presented using a 27-inch monitor with a resolution of 2560 x 1440 pixels and a refresh rate of $60 \mathrm{~Hz}$. The screen was placed $50 \mathrm{~cm}$ from the animal nose. We used alternating grating, horizontal black and white sinusoidal bars alternating at $1 \mathrm{~Hz}$, with fixed spatial frequency of 0.05 cycles/degree and $100 \%$ contrast $^{17}$. Stimuli duration was $2 \mathrm{~s}$, presented at $2.5 \mathrm{~s}$ inter-stimulus-interval. The whole protocol lasted 6 minutes. In addition, for Visual Steady State stimulation, we used flickering light flashes at different frequency $(8,15$ and $30 \mathrm{~Hz})$ presented in trains of $1.2 \mathrm{~s}$, with $2 \mathrm{~s}$ inter-stimulus-interval. This protocol lasted 15 minutes.

\section{EEG recordings in humans}

Participants. We analyzed the EEG recordings from SYNGAP1-ID and neurotypical participants reported $\mathrm{in}^{26}$. Experimental procedures involving humans' subjects were approved by the ethics, scientific and administrative committees of CHU Sainte-Justine. Written informed consent from parents or caregivers and participants' assent were obtained before study participation. Data were collected from 8 participants diagnosed clinically with SYNGAP1 haploinsufficiency and 49 agematched neurotypical (NT) control subjects. Three to seventeen years old participants of both sexes were recruited. Only NT subjects without a family history of neurodevelopmental disorders such as ID or ASD were recruited. Exclusion criteria for all groups consisted of having undergone neurosurgery and/or having vision or hearing problems. Comprehensive clinical phenotype, inclusion criteria and pharmacological treatment of the clinical group is reported $\mathrm{in}^{26}$.

EEG recordings and sensory stimulation protocols. Recordings are described in ${ }^{26}$ and briefly summarized below. EEG recordings took place in an electrically shielded and sound-attenuated room in CHU Sainte-Justine. The recording was carried out using Geodesics 128 electrode nets (Electrical Geodesics System Inc., Eugene, OR, USA). Data was acquired using an EGI Net Amp 300 amplifier and stored on a G4 Macintosh computer with NetStation EEG Software (Version 
4.5.4). The EEG procedure was heavily adapted to the clinical populations in order to increase acceptance. Pictograms and videos were used to prepare participants before coming in for the evaluation and storytelling was used to make the wearing of the EEG net more desirable. A movie was shown during the net installation to increase participants' collaboration. Lights were turned off during the recording session. Auditory stimuli were presented via two lateral speakers (BX5a, M-Audio ${ }^{\circledR}$ BX studio, Cumberland, RI, USA) at a distance of 30 centimeters from participants’ ears and visual stimuli were presented on a computer monitor, at a viewing distance of $60 \mathrm{~cm}$. Stimuli were generated by a Dell Optiplex 790 PC using E-Prime 2.0 (Psychology Software Tools, Inc., Pittsburgh, PA, USA). EEG was recorded at a sample rate of $1000 \mathrm{~Hz}$ using the vertex electrode as online reference. Impedances were kept below $40 \mathrm{k} \Omega^{27}$. During the white noise task and the resting state recording, a silenced movie without subtitles was presented to ensure maximal collaboration. Participants were told to watch the movie and listen passively to the sounds.

We selected three regions of interest (ROI) according to literature of sensory processing in EEG. Auditory processing is reported to peak in vertex and midline electrodes ${ }^{28,}{ }^{29}$, thus we selected Midline central $(\mathrm{Cz})$ surrounding electrodes as ROI for our analyses on auditory perception, as the equivalent to the Primary Auditory Cortex in mice (supplementary figure 1). Cz ROI is composed of the five electrodes surrounding $\mathrm{Cz}$. Visual perception is recorded at the occipital region. Occipital ROI is composed of Oz electrode in addition to four surrounding electrodes. A third ROI corresponding to the Posterior Parietal Cortex was used by grouping $\mathrm{Pz}$ electrode and the five surrounding electrodes as indicated in supplementary figure 1. Each ROI was defined as the average of the corresponding cluster of electrodes mentioned above. Finally, $\mathrm{Cz}$ electrode was used as the digital reference and COM electrode as the analog reference.

Baseline (resting) activity was analyzed during the first three minutes of the recording session. During this time, participants were watching a muted film to engage their participation and encourage immobility. The following sensory stimulation protocols were used. White Noise: Auditory stimulation protocol consisted of a $24 \mathrm{~dB} /$ octave white noise burst presented 150 times. Each stimulus lasted 50ms with an inter-stimulus-interval varying between 1200 and 1400ms to avoid habituation. Total task duration was about 4 minutes. Checkerboard: Visual stimulation was a pattern-reversal black and white checkerboard with a spatial resolution set to $2 * 2 \mathrm{~cm}$ and a luminance of $40 \mathrm{~cd} / \mathrm{m} 2$. Stimulation protocol lasted for 4 minutes with a total of 200 repetitions. 
Pattern reversals were done every $500 \mathrm{~ms}$ for the whole duration of the protocol with no inter stimulus interval. A fixation cross was only presented at the beginning of the task in order to attract the subject's visual attention. Habituation Paradigm: audio-visual speech stimuli were used in order to maximize participants' attention ${ }^{26,30}$. The task had in total 96 sequences of four phonemes /aaai/ and /aaaa/ (75\% /aaai/ and 25\% /aaaa/). Male or female faces are presented with their matched phoneme track four times (aaai or aaaa) then a new sequence start with the other sex. Here analysis was conducted only on the auditory responses elicited by the first and second /a/ to assess the most prominent habituation response. The first presentation of /a/ is referred here as $\mathrm{S} 1$ and the second one as $\mathrm{S} 2$.

\section{EEG analysis in mice and humans}

\section{EEG pre-processing}

Mice EEG signal was downsampled to $2000 \mathrm{~Hz}$. Wideband filter $(0.5-1000 \mathrm{~Hz})$ was applied for ictal spike analysis and a narrower passband filter $(0.5-150 \mathrm{~Hz})$ was applied for the rest of analysis. A notch filter $(59.5-60.5 \mathrm{~Hz})$ was also applied to remove residual $60 \mathrm{~Hz}$ power-line noise contamination. Data were then segmented into periods of different length depending on the stimulation protocol: $1400 \mathrm{~ms}$ (500 ms pre- and $900 \mathrm{~ms}$ post-stimulus onset) for auditory oddball protocol, $2500 \mathrm{~ms}$ (1000 ms pre and $1500 \mathrm{~ms}$ post-stimulus onset) for pure tone stimulation, 3000 $\mathrm{ms}$ (1000 ms pre and $2000 \mathrm{~ms}$ post-stimulus onset) for alternating grating and $4000 \mathrm{~ms}$ (1000 ms pre and $3000 \mathrm{~ms}$ post-stimulus onset) for both visual and auditory steady state. Trials containing per sample segments with an intra-channel average higher than 4 times the total trial standard deviation, were tagged, visually inspected, and removed. Only sessions containing more than 50 clean trials were kept for further analyses.

Humans EEG signal pre-processing and segmentation were carried out using BrainVision Analyser Software (Brain Products, Munich, Germany). EEG raw data was filtered with a lower bound of $0.5 \mathrm{~Hz}$ and a high cut-off at $100 \mathrm{~Hz}$ during data acquisition. A selective $60 \mathrm{~Hz}$ notch filter was applied. Twenty-eight electrodes around the neck and the face were excluded from analysis since they contained muscular artifacts and thus were not useful for further analyses. Blink artefacts and remaining muscular artefacts were removed using a semi-automatic independent component analysis $(\mathrm{ICA})^{31}$. Data were re-referenced to an average reference. For ERP analysis, data was segmented from -200 to $500 \mathrm{~ms}$ relative to stimulus onset and the signal was baseline corrected 
from -150 to 0 ms. For inter-trial coherence (ITC) and time-frequency spectrograms (TFS), epochs were created -800 to $1000 \mathrm{~ms}$ relative to stimulus onset. TFS were baseline corrected from -500 to $0 \mathrm{~ms}$.

The semi-automatic artefact rejection procedure marked segments with voltage exceeding two standard deviations or -200 to $200 \mathrm{uV}$ of amplitude range, which were rejected during the following manual artefact rejection in addition to other segments that were judged noisy. Following artifact rejection, for the white noise paradigm, an average of 139.91/ $150(\mathrm{SD}=11.85)$ epochs were kept for final analysis in control participants, and 105/ 150 (SD = 35.94) for SYNGAP1-ID participants. For the habituation paradigm, an average of 64.22 / 80 (SD = 14.52) epochs were kept for control and an average of 52.17/ $80(\mathrm{SD}=21.19)$ for SYNGAP1-ID participants for both S1 and S2. For the checkerboard paradigm, an average of 126.15/ 200 (SD = 43.48) epochs were kept for the control participants and an average of 81.83/ $200(\mathrm{SD}=35.88)$ epochs for SYNGAP1-ID participants. All epochs were ictal free.

\section{Data Analysis}

Mice and human data were analyzed using the same algorithms. None of the recorded animals were excluded from the final analysis. Signal analysis and quantification was performed using custom MATLAB (The Mathworks Inc., Natik, MA, USA) code, available upon request.

Interictal spikes detection in mice. For each animal, a 2 hours-long epoch was acquired between 10 AM and 4 PM. We then analyzed the first 1 hour of EEG recording performed when mice were freely moving and awake in the open box after the habituation period. We compared rates of interictal spikes and rates of interictal spikes with high frequency oscillations (HFOs) between groups. Processed data were then exported to Matlab R2020a (The Mathworks, MA, USA) and analyzed off-line. Interictal spikes were detected based on threshold crossings (mean and standard deviation), calculated over the entire period. Events above 4 SD were considered as potential interictal spikes. Every detected event was then analysed visually, and false positives caused by movement artefacts were removed manually. Only events detected during periods of immobility $(<2.5 \mathrm{~cm} / \mathrm{s})$ are shown in Figure 1 and Supplemental Figure 2.

Visual Evoked Potential (VEP) and Auditory Evoked Potential (AEP) in mice and humans. EEG signal was low pass filtered to $150 \mathrm{~Hz}$, baseline corrected to the mean voltage of the $150 \mathrm{~ms}$ prior stimulus onset and averaged over trial. ERP components baseline-to-peak N1 and P1 were analyzed 
from AEP and VEP. N1 amplitude was automatically detected by subtracting the minimum voltage (negative peak) within a 10- to 80-millisecond time window after stimulus onset to the averaged baseline value. P1 amplitude was extracted by subtracting the maximum voltage (positive peak) within an 80- to 150-millisecond time window after stimulus onset to the averaged baseline value. Analyses were done using Fieldtrip toolbox v $202009^{32}$.

Habituation and Mismatch negativity (MMN). For quantifying habituation in mice and humans, we calculated the ratio between the amplitudes of the first standard sound (S1) and the second (S2). These ratios were then converted to logarithmic based 10 scale. Mismatch negativity was calculated by subtracting the EEG trace of the standard sound to the EEG trace of the deviant sound. The obtained pattern was then used to detect N1 and P1 amplitudes in each subject.

Power Spectral Density in mice and humans. Resting state data segments were selected with 50\% overlap and a hamming window was applied in order to reduce the amplitudes of the discontinuities at the boundaries of the epochs. Fast Fourier transform was applied using the fft() function available in MATLAB.

Time Frequency Spectrogram (TFS) in mice and humans. TFS analysis allows to explore the power of different frequency bands over time ${ }^{33,34}$. We used complex Morlet's wavelet transformation $^{35}$ to provide power maps in time and frequency domains. The simplified mathematical expression for applying this specific wavelet convolution is:

$$
M(t, f)=W * S=\int_{t} W\left(\frac{t-a}{b}, f\right) S(t) \cdot d t
$$

$M(t, f)$ is a matrix of complex values (vectors) for a given time (t) and frequency (f). $S$ is the signal as a function of time (t) and W corresponds to Morlet's wavelet which is a complex exponential (Fourier) with a Gaussian envelope which undergoes a series of translations (a) and dilations (b) dependently of the frequency (f). Wavelet cycles increase linearly with frequency, beginning at $2 \mathrm{~Hz}[2,0.5]$. Baseline correction was applied using the $500 \mathrm{~ms}$ prior the stimuli onset. This analysis was performed using EEGLAB toolbox (v.13.6.5b) (La Jolla, CA, USA) ${ }^{36}$, in particular using the function newtimef.

Inter-trial coherence (ITC). Analogous to PLV (Phase-locking value), ITC allows the assessment of the strength of phase coherence across trials in temporal and spectral domains ${ }^{34}$. The ITC 
computation uses only the phase of the complex values given by Morlet's wavelet transform. ITC measures phase coupling across trials at all latencies and frequencies and is defined by:

$$
I T C_{t}=\frac{1}{N}\left|\sum_{n=1}^{N} \exp (j \theta(f, t, n))\right|
$$

where $j \Theta(f, t, n)$ represents the phase for a given frequency (f), time point (t) and trial (n). The obtained values are always defined between 0 and 1 . Phase-locking values close to 1 indicate strong inter trial phase-locking, thus representing evoked activity while scores closer to 0 indicate a high inter trial phase variability ${ }^{33,37}$.

Phase Amplitude Coupling (PAC). PAC analysis allows the evaluation of nesting oscillations, where the phase of the lower frequency (here called fp) modulates the amplitude of the higher frequency (fa). The bandwidth of the filter used to obtain 'fp' and ' $\mathrm{fa}$ ' is a crucial parameter in calculating $\mathrm{PAC}^{38}$. The filters for extracting 'fa' need to be wide enough to capture the center frequency \pm the modulating fp. If this condition is not met, then PAC may not be detected even if present $^{39}$. We therefore decided to use a wide enough bandwidth for both ' $\mathrm{fa}$ ' and ' $\mathrm{fb}$ '. The coupling between 'fp' and 'fa' was quantified using the mean vector length modulation index $(\mathrm{MVL}-\mathrm{MI})^{38}$ as it has been suggested to be more sensitive to coupling strength and width compared to other methods ${ }^{39}$. This approach estimates PAC from a signal with length $\mathrm{N}$, by combining phase $(\phi)$ and amplitude information to create a complex-valued signal.

$$
M I=\left|\frac{1}{N} \sum_{t=1}^{N} a_{t} e^{i \theta_{t}}\right|
$$

where $\mathrm{N}$ is the total number of data points, $\mathrm{t}$ is each time point, at is the amplitude of ' $\mathrm{fa}$ ' at the time point $\mathrm{t}$ and $\theta_{\mathrm{t}}$ is the phase angle of 'fp' at time point $\mathrm{t}$.

To evaluate whether the observed modulation index actually differs from what would be expected by chance, surrogate analysis needs to be performed. To do so, surrogate data, are created by shuffling trial and phase-carrying information (500 surrogates), to normalize MI-values. These random permutations create a new time series with broken temporal relationships between the phase and amplitude information. Then MI is estimated again using the shuffled time series to obtain the null distribution of surrogate modulation index values. A normalized modulation index $\left(\mathrm{MI}_{\text {norm}}\right)$ is then obtained as a $z$-score: 


$$
\text { MInorm }=\frac{M I-\mu}{\sigma}
$$

where $\mu$ and $\sigma$ are the mean and standard deviation obtained from the null distribution. A Bonferroni corrected t-test was then performed and all MI-values exceeding a 5\% significance threshold were grouped into clusters for further statistical analysis by group. This analysis was performed using PACmeg code provided by ${ }^{40}$.

\section{Statistical analysis}

Data were systematically tested for Normal distribution, with the Lilliefors test, which is a modification of the Kolmogorov-Smirnov test recommended for small sample sizes ${ }^{41}$. Homoscedasticity was assessed using the Levene's test. Differences between two groups of normally distributed data with homogeneous variances were analyzed using parametric student's t-test, while not normally distributed data were analyzed with Wilcoxon rank sum test. To evaluate sex effect, 2-ways ANOVA (F) test were performed. When no sex effect was observed, main effect (genotype) was analyzed using Tukey posthoc test. For the auditory habituation test S2/S1 ratios were converted to logarithmic based 10 scale because distributions were not homogeneous. Paired sample test was performed using paired T-test when normality was fulfilled, and Wilcoxon signed rank test when it was not. Significance level was set to $5 \%(p=0.05)$. Results were considered significant for values of $\mathrm{p}<0.05$. Data are presented as mean \pm standard error of mean.

\section{Data availability}

All data are available upon reasonable request.

\section{Results}

\section{Syngap 1 ${ }^{+/-}$mice show ictal activity in multiple cortical regions during immobility}

Most SYNGAP1-ID patients have epilepsy ${ }^{2-8,42}$. Interestingly, previous studies reported increased frequency of interictal spike events and occasional electrographic seizures in two different mouse models of Syngapl haploinsufficiency ${ }^{14,15,43}$, which appeared to be most frequent during non-rapid eye movement sleep ${ }^{14,15}$. Interictal spikes are pathological electrical events that reflect seizure susceptibility in epileptic patients ${ }^{44}$. However, since EEG studies had not been previously performed in the Syngapl heterozygous mice line used in our study, if and whether they show interictal spike was unknown. Therefore, as first step we performed video-EEG recordings on freely moving mice in an open field and analyzed interictal spikes during immobility. We observed 
frequent interictal spikes, both sparse and in clusters, in all the recorded Syngap1 ${ }^{+/-}$mice independent of the recorded regions (Fig.1). During the recordings, we observed only one electrographic seizure in one Syngap1 ${ }^{+/-}$mouse, which was not accompanied by clear motor manifestations. We further compared the frequency of interictal spikes in males and females and found that it was independent of the sex (Supplemental figure 2). These results demonstrate that high-amplitude, pathological interictal spikes, in particular during immobility, are a reproducible biomarker across different Syngapl haploinsufficient mouse lines ${ }^{14,15}$.

A

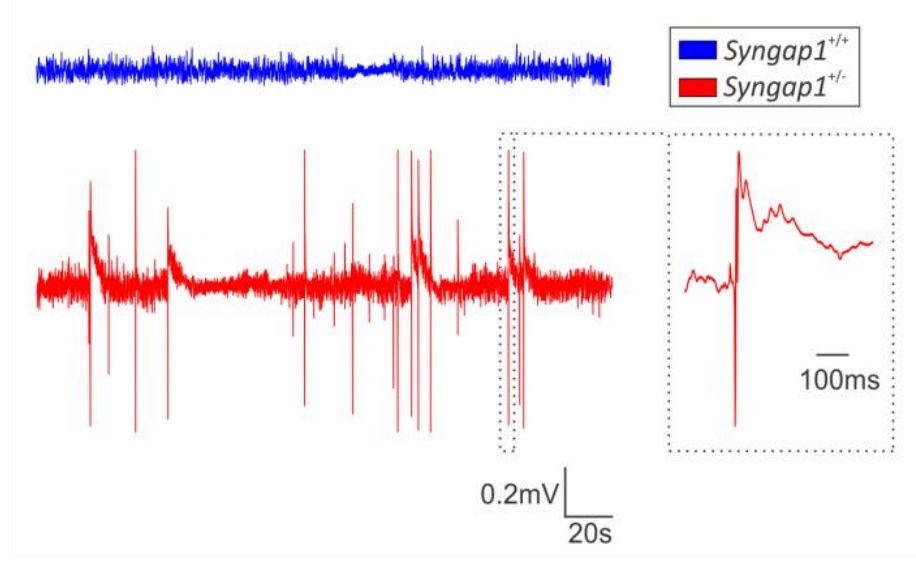

B

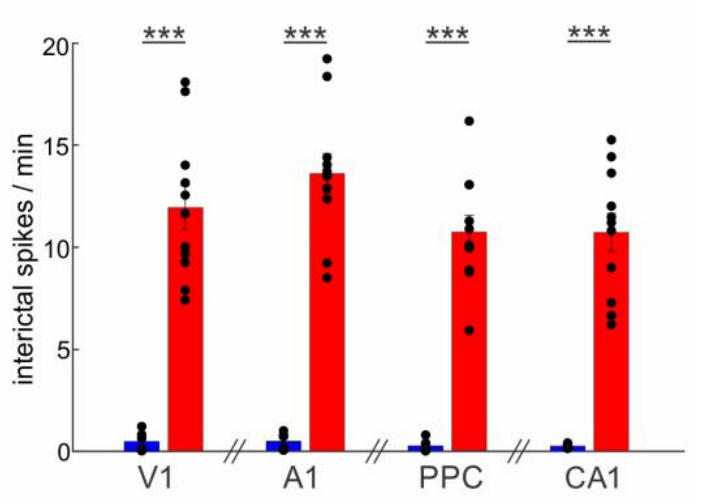

Figure 1. Syngap1 haploinsufficient mice show increased inter-ictal spike activity. A: Example of EEG trace recorded from of a Syngapl ${ }^{+/}$(blue) and Syngap1 ${ }^{+/}$(red) mouse during 2 minutes of immobility/sleep. On the right, interictal spike on an expanded time scale. B: Bar plots show the total number of interictal spikes detected in V1 (t-test $\mathrm{t}(15)=-7.6610, \mathrm{p}<0.0001)$, A1 (t-test; $\mathrm{t}(15)=-9.9019$, $\mathrm{p}<0.0001)$, PPC (t-test; $\mathrm{t}(15)=-9.3477, \mathrm{p}<0.0001)$ and CA1 (t-test; $\mathrm{t}(15)=-7.4481, \mathrm{p}<0.0001)$. *** indicates $\mathrm{p}<0.0001$. Bar graphs represent mean \pm SEM. Syngap ${ }^{+/+} \mathrm{n}=7 ;{\text { Syngap } 1^{+/} \mathrm{n}=11 \text { mice. }}$.

\section{Increased baseline gamma power is a common phenotype in both Syngap1 ${ }^{+/}$mice and SYNGAPI-ID patients}

Neural oscillations in the gamma band $(35-100 \mathrm{~Hz})$ are associated with sensory processing and $\operatorname{cognition}^{45}$. Remarkably, abnormal gamma oscillations have been reported in a variety of neuropsychiatric and neurodevelopmental disorders, including $\mathrm{ASD}^{46-49}$ and schizophrenia ${ }^{50-52}$. Here, we performed power density spectra analysis on 3-minutes EEG periods during baseline activity (mice were freely exploring the Open Field but no stimulus was presented) in Syngap1 ${ }^{+/+}$ and Syngap1 ${ }^{+/-}$mice and quantified mean values for beta $(14-30 \mathrm{~Hz})$, low gamma $(35-55 \mathrm{~Hz})$ and high gamma $(65-100 \mathrm{~Hz})$. Syngap $1^{+/-}$mice showed a statistically significant increase in low gamma 
and high gamma power compared to wild-type littermates, which was consistent in all the recorded regions (Figure 2A, B). Both females and males Syngap1 ${ }^{+/-}$mice showed increased gamma power, although appeared to be more pronounced in males (Supplementary figure 3).

Further, to investigate whether SYNGAP1 haploinsufficiency is also associated with increased baseline gamma power in humans, we performed the same analysis as we did in mice using EEGs previously recorded from SYNGAP1-ID individuals ${ }^{26}$. Similar to what was observed in the mouse model, we found a statistically significant increase in both low and high gamma bands, consistent across all regions of interested (Figure 2C, D), during baseline activity. We further observed a significant increase in beta power in SYNGAP1-ID patients which was not present in Syngap1 ${ }^{+/-}$ mice (Figure 2B, D). These data suggest that increased power of the baseline (non-stimulus) gamma band is a robust and translational biomarker for Syngap1/SYNGAP1 haploinsufficiency.

A
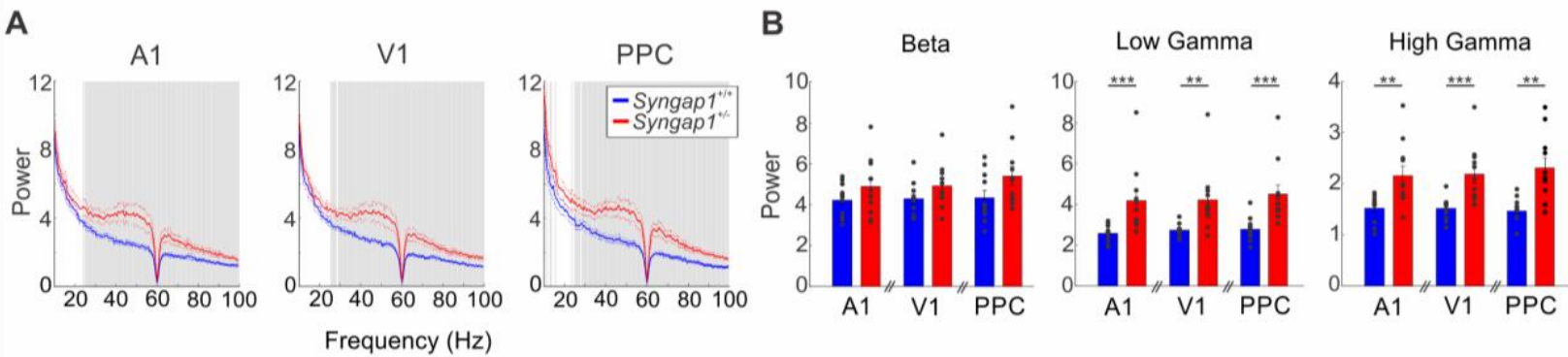

$c$
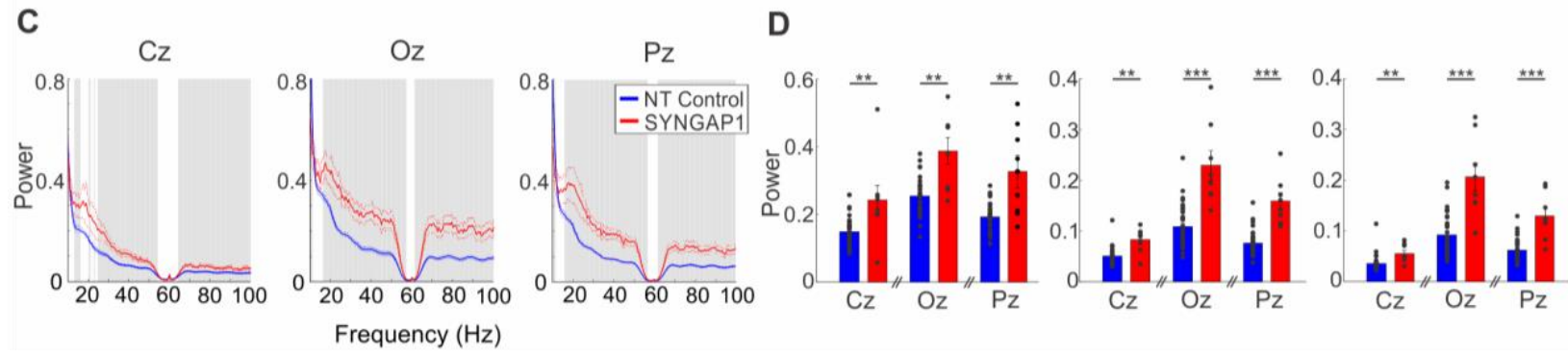

Figure 2. Syngap1/SYNGAP1 haploinsufficiency correlates with increased baseline gamma power in both mice and humans during baseline state. A, C: Power Density Spectra of 3-minutes baseline state (non-stimulus) EEG signal from $\mathrm{A} 1 / \mathrm{Cz}, \mathrm{V} 1 / \mathrm{Oz}$ and $\mathrm{PPC} / \mathrm{Pz}$, in mice (A) and humans $(\mathbf{C})$. Gray bars represent significant differences ( $\mathrm{t}$-test, $\mathrm{p}<0.05$ ). B,D, Bar plots shows mean powers \pm SEM of the beta band in mice (A1: Wilcoxon rank sum test; $\mathrm{z}=-1.0506, \mathrm{p}=0.2934 ; \mathrm{V} 1$ : Wilcoxon rank sum test; $\mathrm{z}=-1.5103$, $\mathrm{p}=0.1310$; PPC Wilcoxon rank sum test; $\mathrm{z}=-1.9043, \mathrm{p}=0.0569)$ and humans (Cz: Wilcoxon rank sum test; $\mathrm{z}=-2.7387, \mathrm{p}=0.0062 ; \mathrm{Oz}$ : Wilcoxon rank sum test; $\mathrm{z}=-3.0741, \mathrm{p}=0.0021$; Pz Wilcoxon rank sum test; $\mathrm{z}=-$ 2.7308, $\mathrm{p}=0.0063$ ), low gamma band in mice ( $\mathrm{A} 1$ : Wilcoxon rank sum test; $\mathrm{z}=-3.3489, \mathrm{p}=0.0008$; V1 : Wilcoxon rank sum test; $\mathrm{z}=-3.2176, \mathrm{p}=0.0013$; PPC Wilcoxon rank sum test; $\mathrm{z}=-3.4802, \mathrm{p}=0.0005)$ and humans (Cz: Wilcoxon rank sum test; $\mathrm{z}=-2.9949, \mathrm{p}=0.0027$; Oz : Wilcoxon rank sum test; $\mathrm{z}=-3.9480$, $\mathrm{p}=0.00007$; Pz Wilcoxon rank sum test; $\mathrm{z}=-4.0728, \mathrm{p}=0.00004$ ), and high gamma band in mice (A1: 
Wilcoxon rank sum test; $\mathrm{z}=-3.0206, \mathrm{p}=0.0025 ; \mathrm{V} 1$ : Wilcoxon rank sum test; $\mathrm{z}=-3.4802, \mathrm{p}=0.0005$; PPC Wilcoxon rank sum test; $\mathrm{z}=-3.2176, \mathrm{p}=0.0013)$ and humans $(\mathrm{Cz}$ : Wilcoxon rank sum test; $\mathrm{z}=-3.1551$, $\mathrm{p}=0.0016$; Oz : Wilcoxon rank sum test; $\mathrm{z}=-3.7295$, $\mathrm{p}=0.00019 ; \mathrm{Pz}$ Wilcoxon rank sum test; $\mathrm{z}=-3.6983$, $\mathrm{p}=0.00021)$. Note significantly increased power of low and high gamma band $(* * \mathrm{p}<0.01 ; * * * \mathrm{p}<0.001)$ in mice and humans. Number of mice: Syngap $1^{+/+} \mathrm{n}=11 ;$ Syngap $^{+/-} \mathrm{n}=11$; number of human participants: NT Controls n=35; SYNGAP 1 -ID n=8.

\section{Syngap1/SYNGAP1 haploinsufficiency in associated with excessive theta/gamma phase- amplitude coupling following visual and auditory stimulations}

Sensory-evoked potentials induced by simple sensory stimulus, in particular visual-evoked potentials (VEPs) and auditory-evoked potentials (AEPs) described by the waveform of EEG activity in the first $500 \mathrm{~ms}$ after stimulus presentation, are the cortical responses most commonly used to study sensory perception in both humans and mouse models ${ }^{16}$. They been proposed as biomarkers for several neurodevelopmental disorders, including $\mathrm{ASD}^{16,17,20,53,54}$. Here, we analysed VEP and AEP amplitudes in Syngap1 ${ }^{+-}$mice and control littermates (Figure 3). Similar to published reports from Rett's syndrome patients and mouse models ${ }^{17}$, Syngap1 ${ }^{+/}$mice exhibited reduced amplitude of the P1 component of the VEPs compared to wild-type mice (Figure 3A). On the other hand, AEPs analysis showed increased N1 amplitude but no change in P1 in the Syngap1 ${ }^{+/}$mice (Figure 3C). For both VEPs and AEPs, we observed a significant effect of the Syngapl genotype, but not sex-related differences, and no statistical significant interaction was detected between these two factors (Supplementary Figure 4).

Next, we performed the same analyses on EEGs recorded from SYNGAP1-ID patients. Consistent with what we found in mutant mice (Figure 3A), we observed reduced P1 amplitude in VEPs recorded from SYNGAP1-ID patients as compared to the corresponding control group (Figure 3B). In contrast, AEP analysis highlighted different alterations within Syngap1 ${ }^{+/-}$mice and SYNGAP1ID patients. SYNGAP1-ID patients exhibit reduced P1, with no change in N1 (Figure 3D), while Syngap ${ }^{+/-}$mice showed the opposite (compare Figure 3C and 3D).

Our results indicate that abnormalities in sensory-evoked related potentials do not provide a translationally robust biomarker for SYNGAP1/Syngapl haploinsufficiency, since they may be species-specific as well as dependent on the specific sensory modality. 
Mouse

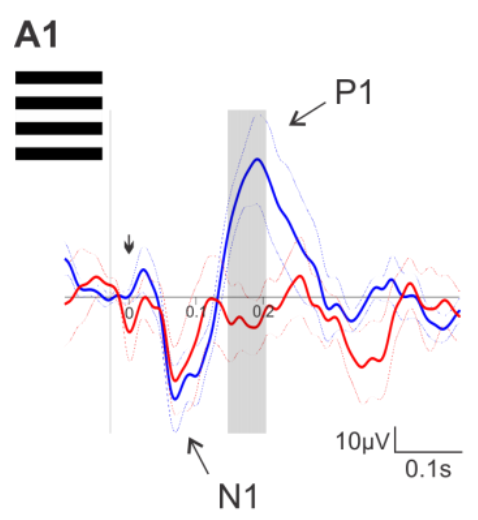

\section{A2}

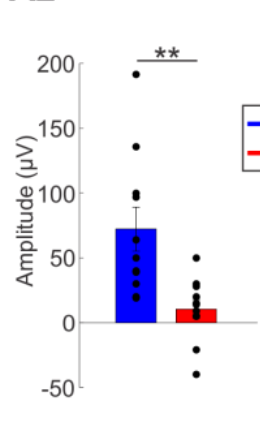

C1

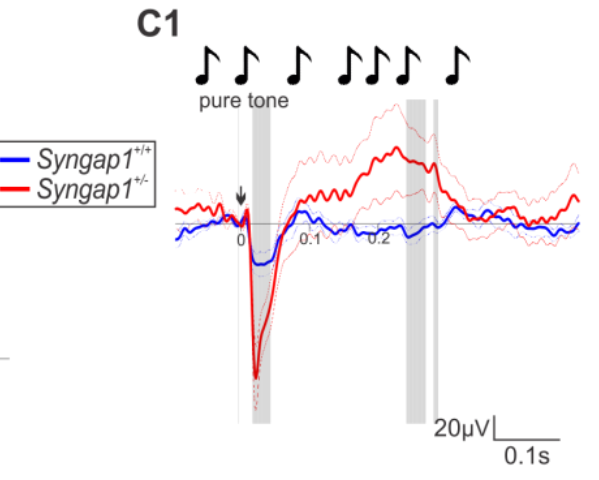

Human

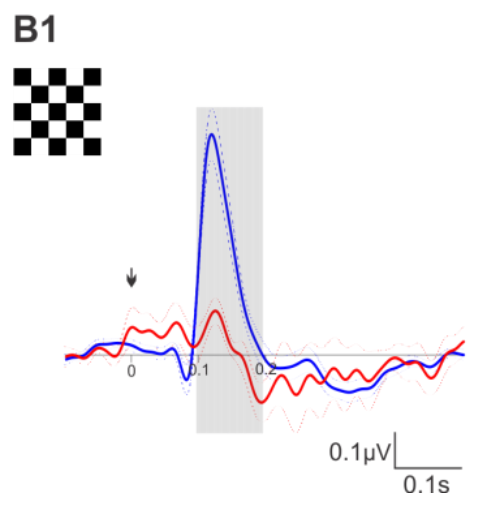

\section{B2}

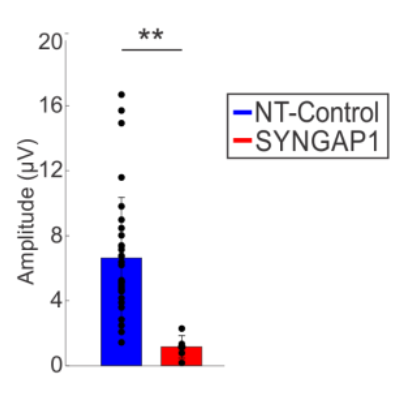

D1

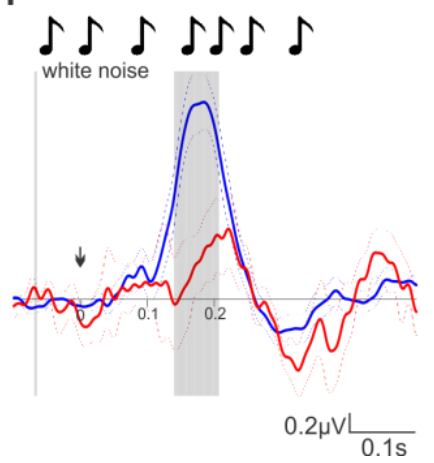

C2

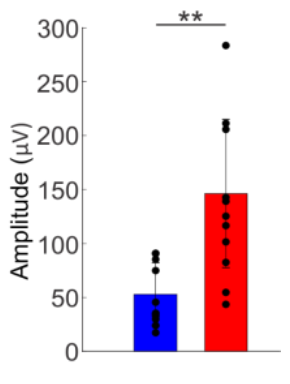

Figure 3. Syngap1 haploinsufficiency correlates with decreased responses to visual stimuli in both patients and mice, while alterations of auditory responses are species-specific. A1-B2: VEP responses in mice (A1, A2) and humans (B1, B2). A1, B1: Grand average traces. Arrows show the start of stimulation. Shadow gray bars represent significant differences $(\mathrm{p}<0.05)$ between groups A2, B2: Bar plots shows P1 amplitude in (A2) mice (Wilcoxon rank sum test; $\mathrm{z}=-3.1884, \mathrm{p}=0.0014$ ) and (B2) humans (Wilcoxon rank sum test; $\mathrm{z}=3.7430, \mathrm{p}=0.0001)$. C1-D2: AEP response in mice $(\mathrm{C} 1, \mathrm{C} 2)$ and humans (D1, D2). Syngap $1^{+/+} \mathrm{N}=11 ;$ Syngapl $^{+/} \mathrm{n}=11$ mice, and NT Controls n=26; SYNGAP1-ID n=7 human participants. C1, D1: Grand average traces. C2: Bar plot shows N1 amplitude in mice (Wilcoxon rank sum test; z=3.0863, p=0.0020). D2: Bar plot shows P1 amplitude in humans (Wilcoxon rank sum test; $\mathrm{z}=1.9899$, $\mathrm{p}=0.0474)$. Bar graphs represent mean \pm SEM. Syngap ${ }^{+/+} \mathrm{n}=11$ and Syngap ${ }^{+/} \mathrm{n}=11$ mice; NT Controls $\mathrm{n}=29$ and $S Y N G A P 1-\mathrm{ID} \mathrm{n}=8$.

Since the analysis of sensory-evoked local field potential amplitudes or delays did not show consistent differences across species (human and mice) and sensory perception modalities (auditory and visual), we decided to perform more sophisticated analyses. First, we asked whether there could be genotype-dependent effects on the temporal changes in distinct oscillation power over the frequency domain. Time frequency spectrograms (TFS) showed significantly different 
patterns between wild-type/neurotypic and Syngap1/SYNGAP1 individuals (Figure 4, A1-D1). Although we could not identify a motif common to both mice and humans in any of the tested sensory modalities, we noted that gamma power seemed to be temporally organized by a lower rhythm in the theta band. To further investigate this possibility, we performed phase amplitude coupling (PAC) analysis. We found significantly increased PAC between theta and gamma bands, in both Syngap1 1/- mice and SYNGAP1-ID patients, which was further consistent across sensory modalities (Figure 4A2-D2), suggesting that sensory stimulations lead to excessively coordination of the temporal organization of oscillatory activity in individuals carrying Syngap1/SYNGAP1 pathogenic variants. Altogether, these results demonstrate that increased theta/gamma PAC evoked by sensory stimulation is a strong, reproducible biomarker of Syngap1/SYNGAP1 haploinsufficiency. 


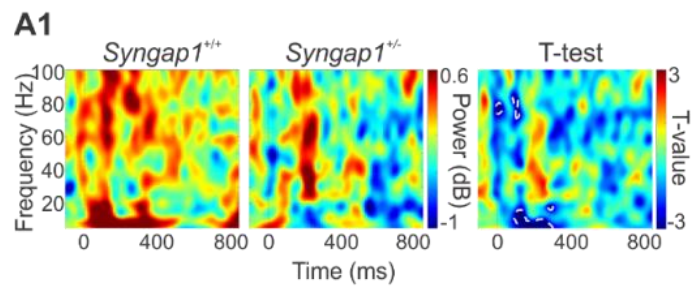

Human

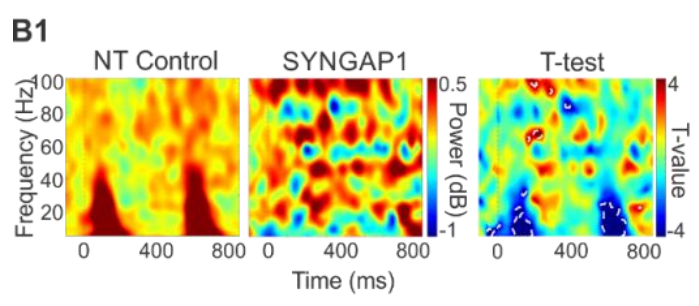

Mouse

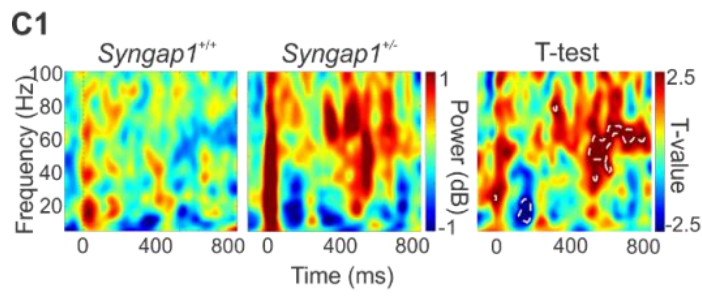

Human

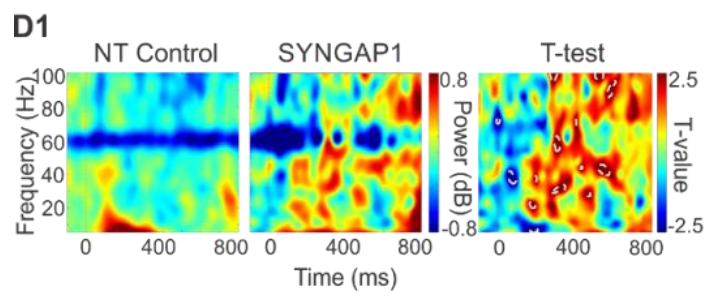

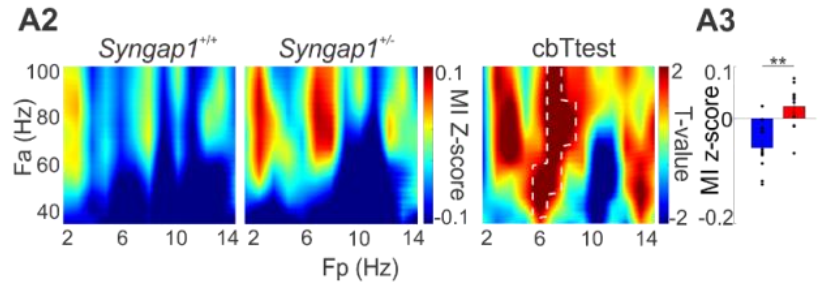

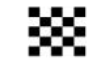

B2
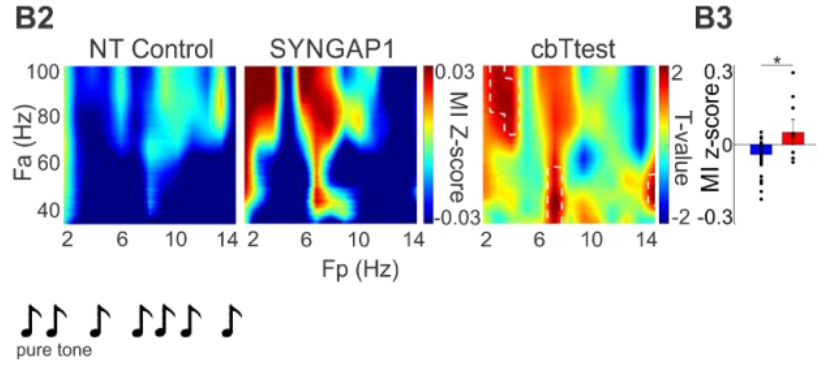

C2

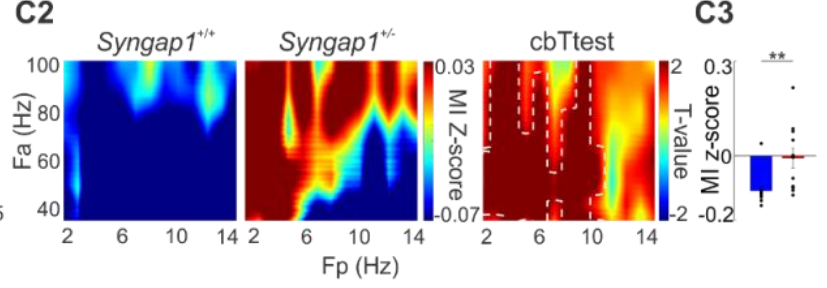

Shite noise 5525

D2
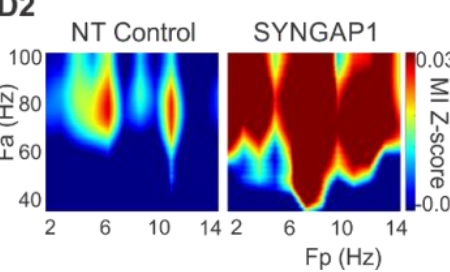

cbTtest

D3

Figure 4. Theta/gamma phase-amplitude coupling (PAC) following auditory and visual stimuli is significantly increased in both Syngap ${ }^{+/-}$mice and SYNGAP1-ID patients. A1-D1: Time Frequency Spectrogram showing visual $(\mathbf{A 1}$, B1) and auditory $(\mathbf{C 1}, \mathbf{D 1})$ evoked responses in mice $(\mathbf{A 1}, \mathbf{C 1})$ and humans (B1, D1). Dotted lines represent statistical differences $(\mathrm{p}<0.05)$ in Welch t-test map (right panels). Note that gamma power seems to be temporally organized by a lower frequency rhythm, which is particularly clear in B1. A2-D2: Phase-amplitude comodulograms and cluster based statistics. Statistical differences $(\mathrm{p}<0.05)$ are marked by white dotted lines. A3-D3, Bar plots shows z-score values corresponding to theta/gamma PAC. A3: t-test, $\mathrm{T}(20)=-4.1000, \mathrm{p}=0.0005 ; \mathbf{B 3}$ : t-test, $\mathrm{T}(20)=-3.1898, \mathrm{p}=0.0033$; C3: Wilcoxon rank sum test; $\mathrm{z}=-3.0863, \mathrm{p}=0.0020$; D3: Wilcoxon rank sum test, $\mathrm{z}=-2.8813, \mathrm{p}=0.0092$; ** indicates $\mathrm{p}<0.01$, * indicates $\mathrm{p}<0.05$. A, C : Syngap $1^{+/+} \mathrm{n}=11 ;$ Syngapl $^{+/-} \mathrm{n}=11$ mice; B: number of human participants, NT Controls n=26; SYNGAPI-ID n=7; D: : number of human participants, NT Controls $\mathrm{n}=29$ ; SYNGAPI-ID n=8. 


\section{Neural entrainment abnormalities are sensory-modality dependent in Syngap ${ }^{+/-}$mice}

When presented with rhythmic sensory stimulations oscillating at a certain frequency, neural networks oscillate in time to the stimulus, a process referred to as entrainment. Neural entrainment is thought to increase the ratio of signal to noise in the local cortical network, thus increasing sensory gain for a specific frequency ${ }^{55}$. Abnormal auditory entrainment following auditory stimulations has been reported in idiopathic ASD, and both in Fragile X syndrome patients ${ }^{56-59}$ and mouse models ${ }^{25}$. To explore whether similar neural entrainment abnormalities are caused by Syngapl haploinsufficiency, we recorded EEGs following repetitive auditory and visual entrainment protocols presented at different frequencies. Inter-trial coherence analysis showed significantly increased auditory entrainment following stimulation with click trains at $40 \mathrm{~Hz}$ (Figure 5B), but not at $10 \mathrm{~Hz}$ (Figure 5A), in Syngap1 ${ }^{+/-}$mice compared to control littermates. In contrast, visual entrainment to stimuli (flickering lights) presented at $8 \mathrm{~Hz}$ (Figure 5C) was significantly reduced in mutant mice. However, visual entrainment following stimulation at $15 \mathrm{~Hz}$ (Figure 5D) or $30 \mathrm{~Hz}$ (data not shown) was indistinguishable in Syngapl ${ }^{+/}$versus control mice. No sex differences were identified in any of the entrainment paradigms (Supplementary Figure 5). 

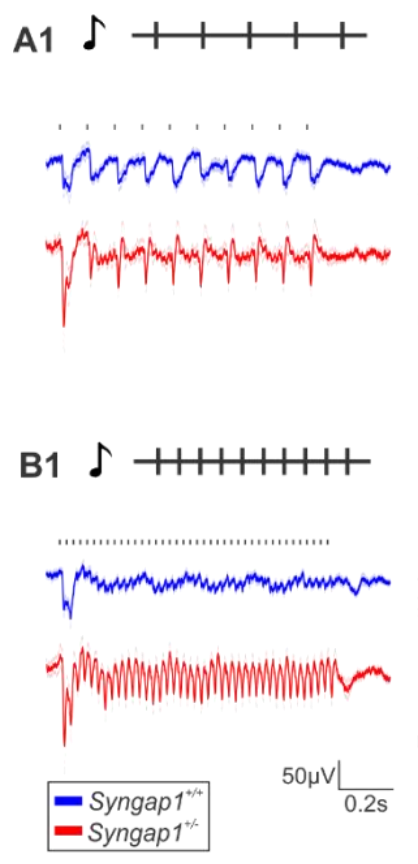

C1

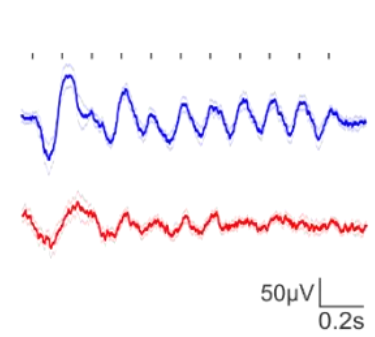

D1
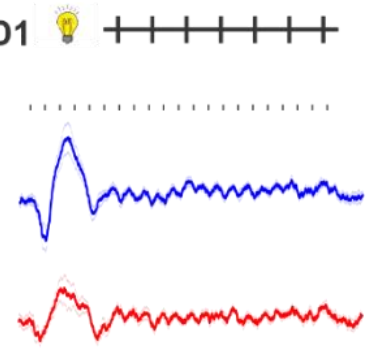

A2

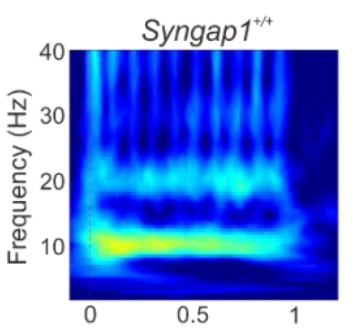

B2

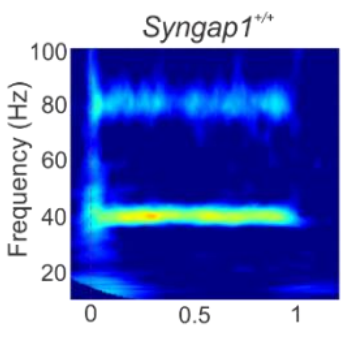

C2

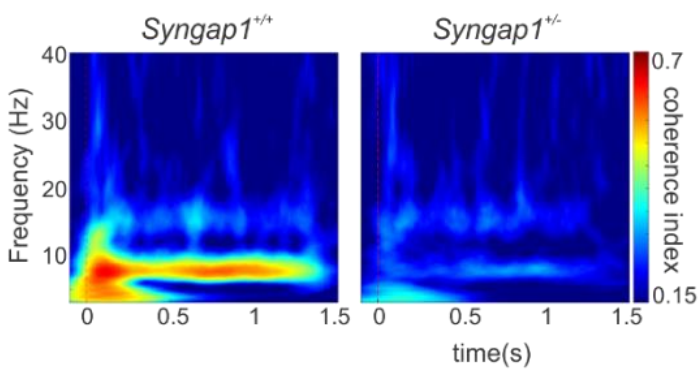

D2
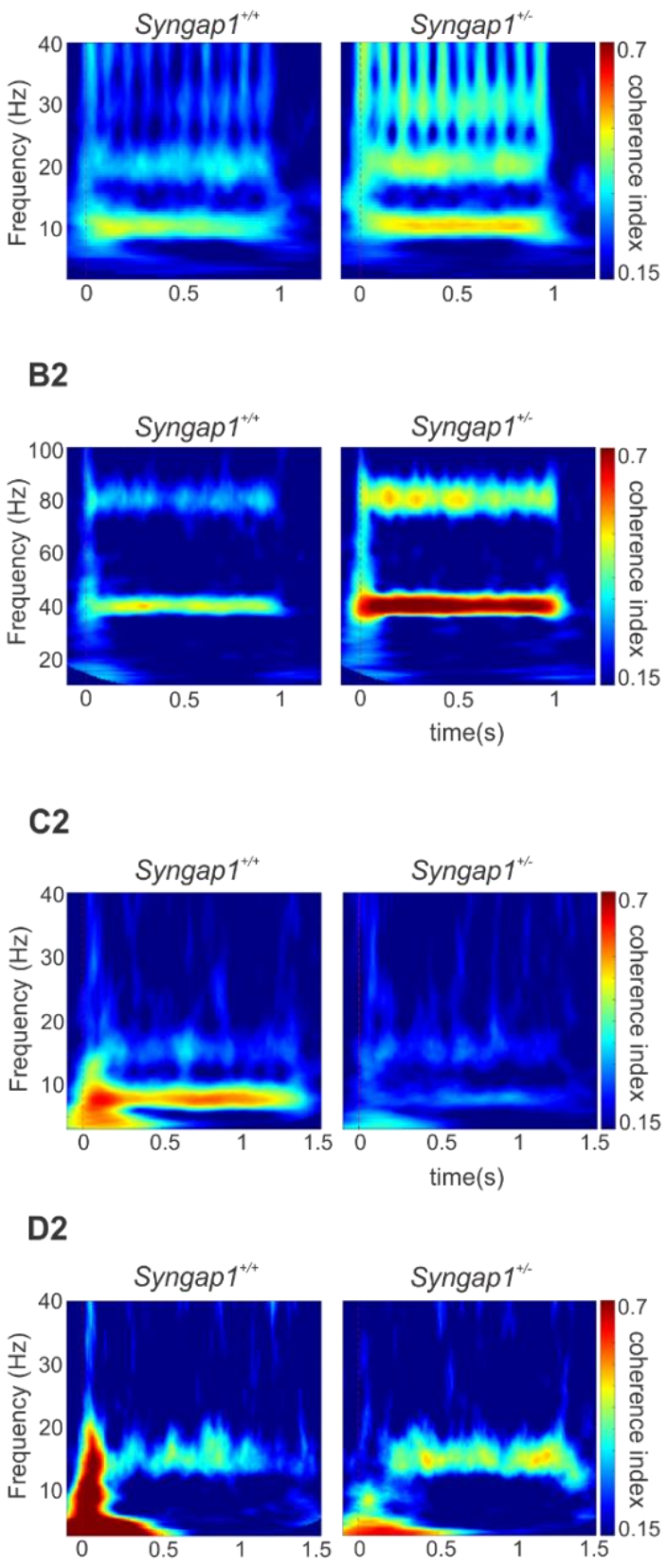
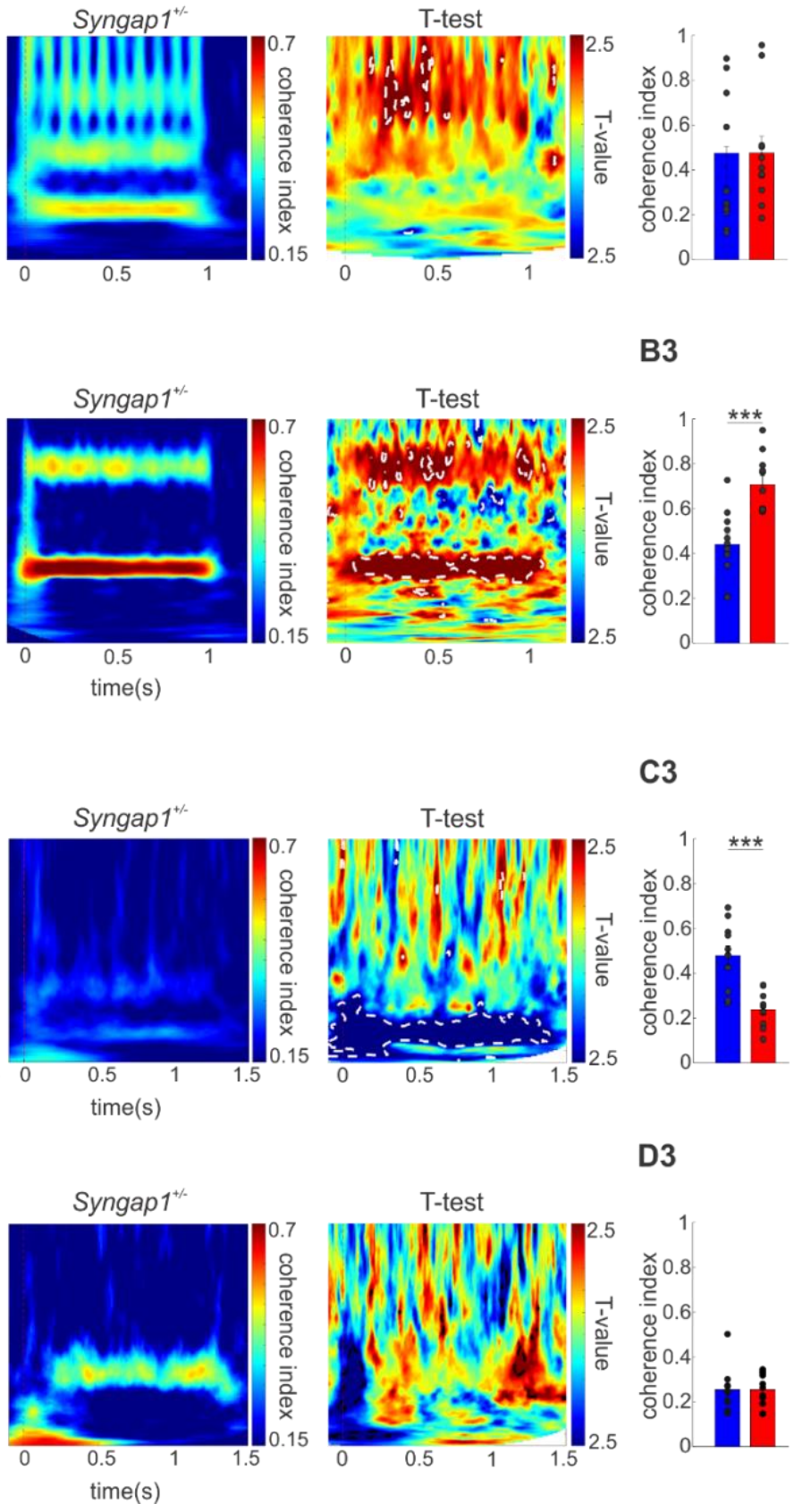

B3

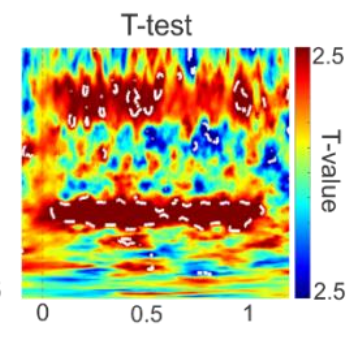

C3

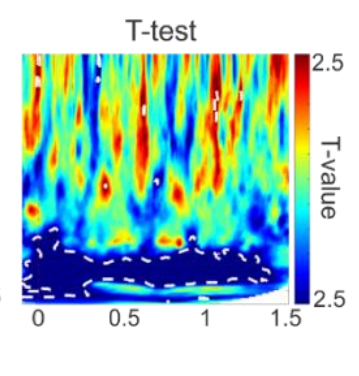

D3

A3
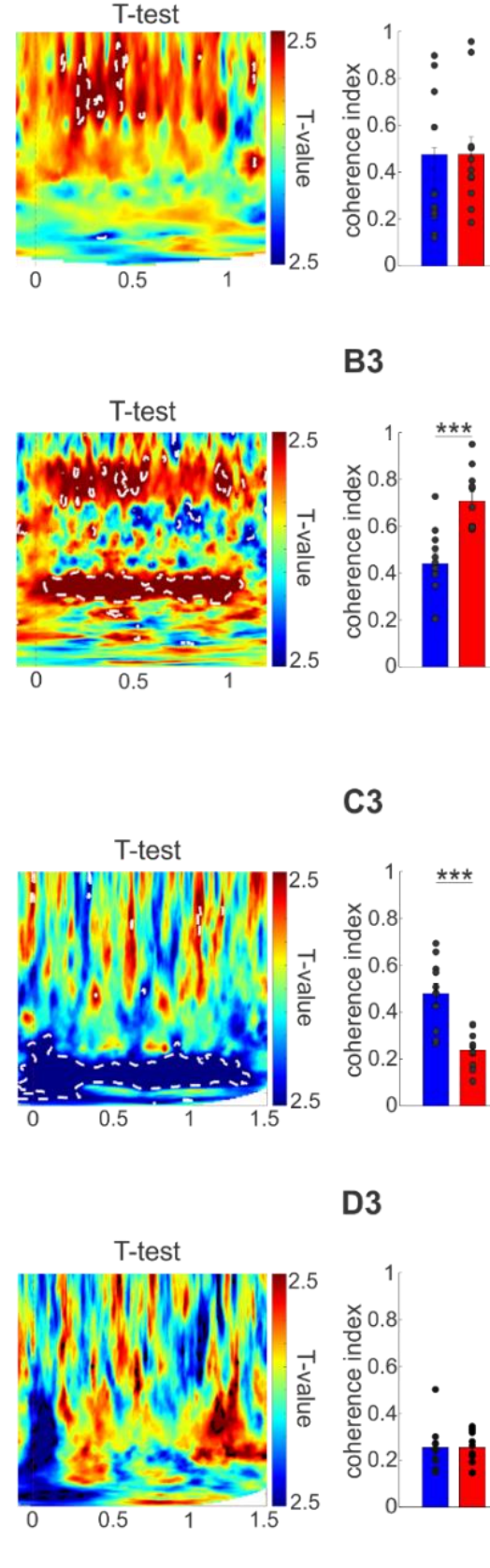

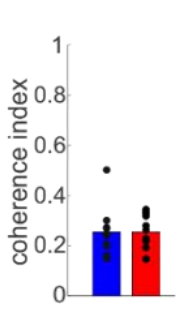

Figure 5. Syngap1 haploinsufficiency in mice is associated with excessive auditory entrainment at $40 \mathrm{~Hz}$ but not at $10 \mathrm{~Hz}$ and reduced visual entrainment at $8 \mathrm{~Hz}$ but not 15Hz. A1-D1: Grand average traces. On top, diagram showing the stimulation protocol: Auditory entrainment at $10 \mathrm{~Hz}$ (A1), at $40 \mathrm{~Hz}$ (B1) and visual entrainment at $8 \mathrm{~Hz}(\mathrm{C} 1)$ and $15 \mathrm{~Hz}(\mathrm{D} 1)$. A2-D2: Inter-trial coherence and Welch t-test maps. Statistical differences $(\mathrm{p}<0.05)$ are marked by white dotted lines. A3-D3: Bar plots shows coherence index values corresponding to the stimulating frequency. A3: T-test, $\mathrm{T}(20)=0.5430, \mathrm{p}=0.5931$; B3: Wilcoxon rank sum test, $\mathrm{z}=-3.5459, \mathrm{p}=0.0003$; C3: Wilcoxon rank sum test; $\mathrm{z}=-3.4146, \mathrm{p}=0.0006$; D3: Wilcoxon rank sum test; $\mathrm{z}=-0.3583, \mathrm{p}=0.7427 * * *$ indicates $\mathrm{p}<0.001$. Syngap ${ }^{+/+} \mathrm{n}=11 ;$ Syngap $^{+/-} \mathrm{n}=11$ mice. 
These results suggest that neural entrainment alterations may be specific not only to particular frequencies but also to sensory modalities. The EEG recordings from SYNGAP1-ID patients that we had access to did not include stimulation protocols aimed at eliciting neural entrainment; however, our results suggest neural entrainment could be a potentially interesting biomarker to be explored in these patients.

Finally, we evaluated the synchronicity of brain oscillations to a chirp-modulated tone, since this stimulus has been reported to be reduced in Fragile X syndrome patients and mouse models ${ }^{25,} 57$, 58. Fragile $\mathrm{X}$ syndrome is a neurodevelopmental disorder, which could potentially share some pathophysiological mechanisms with the Syngapl haploinsufficiency ${ }^{60}$. We tested both up and down chirps to ensure that the differences are specific to modulation frequency and not affected by the direction of frequency change in the sound. In contrast to previous observations in Fragile $\mathrm{X}$ Syndrome, Syngap1 1/- male mice showed an increased response to up (Supplemental Figure 6) and down (data not shown) chirp stimulation. Strikingly, in our experiments, neural responses to a chirp stimulus were sex dependent, since we could not induce neural entrainment with a chirp stimulus neither in wild-type nor in mutant female mice (Supplemental Figure 6). Given the lack of reproducible response to a chirp stimulus in females as compared to males, this particular stimulation paradigm may not be an appropriate translational biomarker.

\section{Syngap1/SYNGAP1 haploinsufficiency in associated with decreased habituation to repetitive auditory stimuli}

Habituation responses to repeating tones (auditory gating) or novelty detection responses (mismatch negativity) have been largely employed as biomarkers in neurodevelopmental disorders $^{61}$. Therefore, we investigated whether Syngap $1^{+/-}$mice show deficits in habituation, by analyzing cortical response to 5-9 consecutive sounds presented at a fixed repetition rate of $1 \mathrm{~Hz}$ (Figure 6A1). In particular, we measured N1 amplitude of AEP evoked by the first (S1) and the second sound (S2). In contrast to the clear reduction of $\mathrm{N} 1$ amplitude observed in Syngap1 ${ }^{+/+}$mice, Syngap ${ }^{+/-}$mice showed no change in N1 amplitude between S1 and S2 (Figure 6 A2, A3), and this phenotype was similar in both sexes (Supplementary Figure 7).

In keeping with our observation in the mouse model, we observed a similar lack of habituation in SYNGAP1-ID individuals (Figure 6B2, B3). Taking into account the difference between absolute 
$\mathrm{N} 1$ amplitudes between different mice or individuals, we calculated the S2/S1 ratio for each subject (Figure A4, B4) and found a consistent lack of habituation in both Syngap1 ${ }^{+/-}$mice and SYNGAP1ID patients, suggesting that it could be a robust translational biomarker for Syngap1/SYNGAP1 haploinsufficiency
A1
Mouse
B1
Human

$\cos 225$

a a a

A2

B2

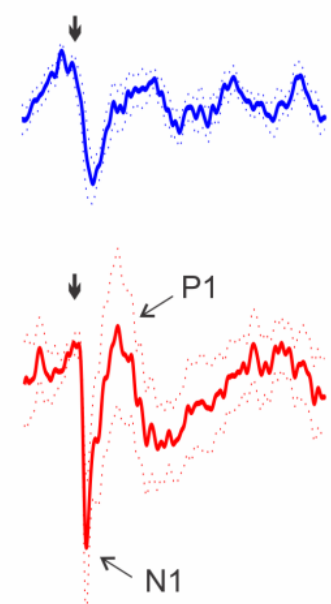

A3

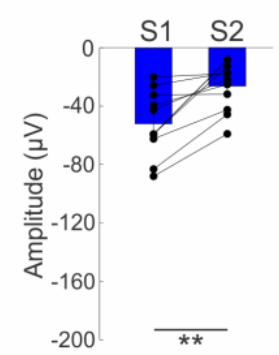

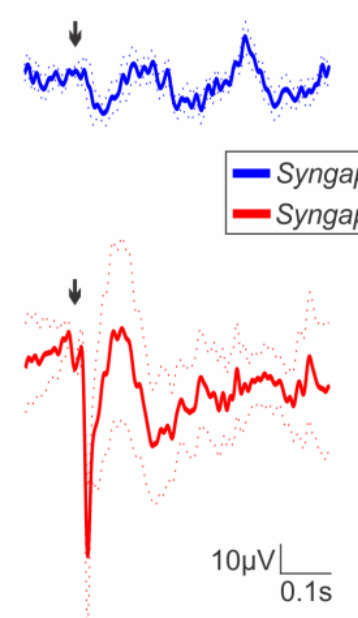

A4

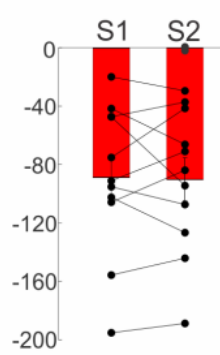

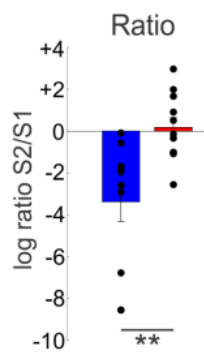

B3
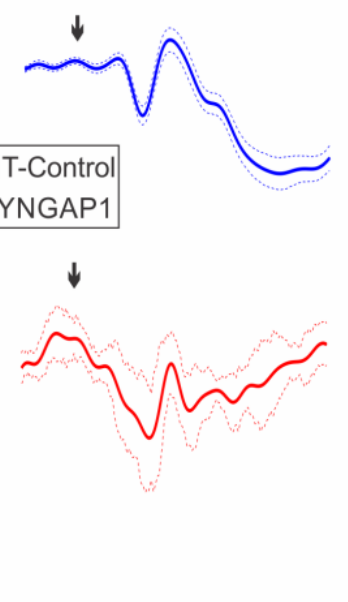
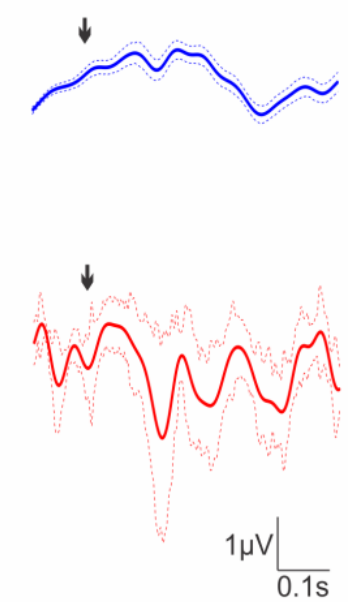

Figure 6. Syngap1/SYNGAP1 haploinsufficiency is associated with a lack of habituation to repetitive sounds in both mice and humans. A1-B2: Schematic of repetitive auditory stimulation protocol in mice (A1) and humans (B1). A2, B2: AEP response to the first (left) and second (right) standard sound of Syngap1 $^{+/+}$(blue) vs Syngap1 ${ }^{+-}$(red) mice (A1) and NT-Controls vs SYNGAP1-ID individuals (B1). A3, B3: Bar plots show P1 amplitude after first (S1) vs second (S2) sounds in mice (A2; Syngap1 ${ }^{+/+}$mice, Wilcoxon signed rank, z=-2.9355, $\mathrm{p}=0.0033$ and Syngap1 ${ }^{+/-}$mice, Wilcoxon signed rank, $\mathrm{z}=0.1778$, $\mathrm{p}=0.8589$ ) and humans (B2; NT-Control individuals, paired t-test, T(48)=-7.6659, p<0.0001 and SYNGAP1ID individuals, paired t-test, T(7)=0.12209, p=0.9141). A4, B4: Logarithmic ratio S2/S1 in mice (Wilcoxon rank sum test; $\mathrm{z}=-3.0214, \mathrm{p}=0.0025$ ) and humans (Welch independent t-test; $\mathrm{T}(14)=0.0282$ ). 
Syngap $^{+/+} \mathrm{n}=11 ;$ Syngap $^{+/-} \mathrm{n}=11$ mice; number of human participants, NT Controls $\mathrm{n}=49 ;$ SYNGAP1-ID $\mathrm{n}=8$.

Mismatch negativity (MMN) is a memory-based brain response to any discriminable change in a stream of auditory stimulation ${ }^{62}$. MMN has recently gained attention as a tool for neurological evaluations since several studies indicate that it is a robust marker of cognitive dysfunction ${ }^{63}$. Here, we explored whether the ability to detect actual physical difference in sounds was altered in Syngap $1^{+/-}$mice, by using a sequence of repetitive, standard sensory stimuli, interrupted by an oddball or deviant stimulus (Figure 7A). We found that the mean amplitude of the first negative peak of MMN (N1), which indicates the sound-specific responses to the auditory stimuli presented, was not statistical different between genotypes (Figure 7C, D). Conversely, while the MMN pattern in wild-type mice showed the expected positive peak at 70-110 ms after the stimulus presentation (P1), indicating that the deviant sound was detected correctly, this peak was wholly absent in Syngap $1^{+/-}$mice (Figure 7C, D). No sex differences were observed for the described MMN pattern (Supplementary Figure 7). Overall, these results suggest that, while responses to frequence specificity are intact at the primary sensory level, deviant sound detection, known to rely on higherorder sensory functions, is specifically altered in Syngap1 ${ }^{+/-}$mice.

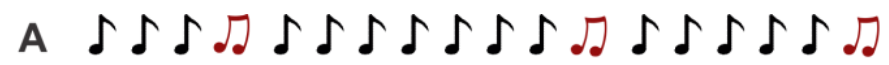

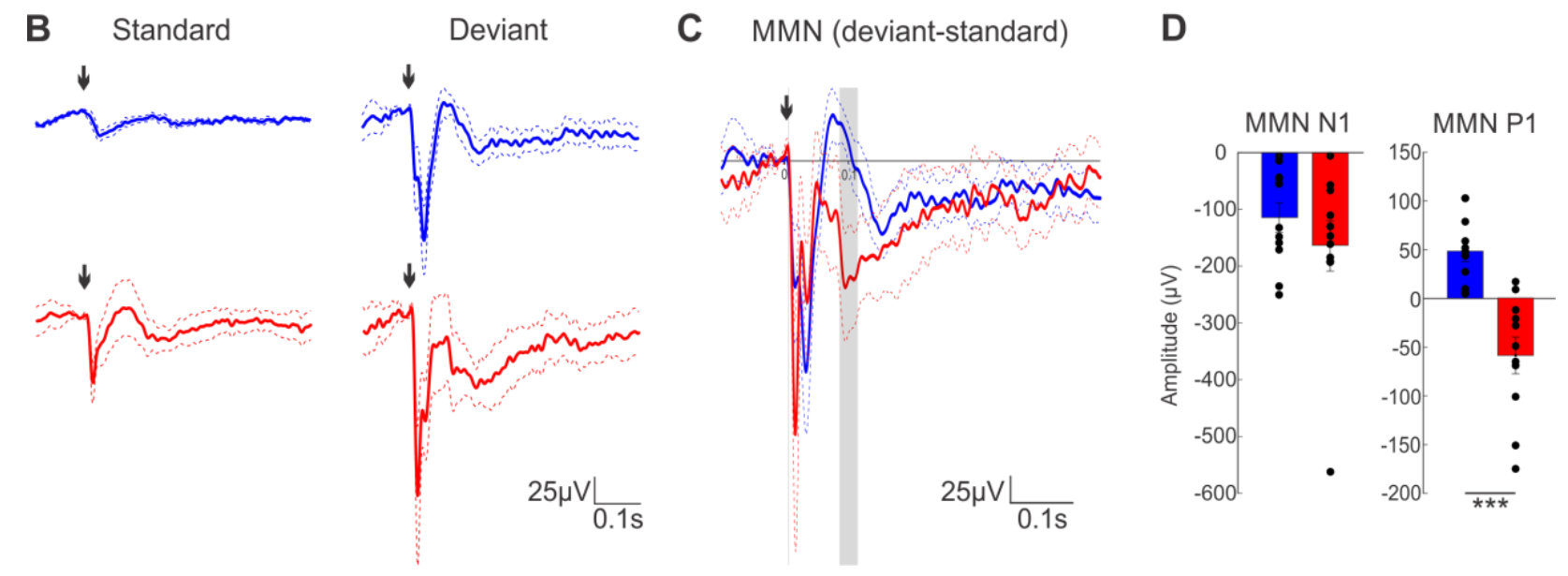


Figure 7. Syngap1 haploinsufficiency is associated with an abnormal MMN pattern in mice. A: Schematic of Oddball stimulation protocol in mice. B: AEP traces of the standard (left) and deviant (right) sounds of Syngap1 ${ }^{+/+}$(blue) and Syngap1 ${ }^{+/-}$(red) mice. C: superposed MMN (deviant - standard) traces. D: Bar plots of MMN N1 (Wilcoxon rank sum test; $\mathrm{z}=-0.7223$, $\mathrm{p}=0.4701$ ) and MMN P1 (Wilcoxon rank sum test; $\mathrm{z}=-3.5469, \mathrm{p}=0.0003)$ peak amplitude. Shadow gray bars represent significant differences $(\mathrm{p}<0.05)$ between groups. Arrows indicate the start time of stimulation. $* *$ indicates $\mathrm{p}<0.01$, *** indicates $\mathrm{p}<0.001$. Syngap $1^{+/+} \mathrm{n}=11 ;{\text { Syngap } 1^{+/} \mathrm{n}=11 \text { mice. }}$

\section{Discussion}

Syngapl haploinsufficiency is associated with intellectual disabilities, developmental delay, epilepsy, poor or absent language development, autistic traits and other behavioral alterations. Sensory processing and perception have been recently shown to contribute to cognitive and behavioral dysfunction in specific neurodevelopmental disorders, including ASD; however, whether and to what extent visual and auditory sensory processing is affected in SYNGAP1-ID or in related mouse models remained unclear. Here, we identified, in Syngap1 ${ }^{+/}$mice, specific alterations in multiple aspects of auditory and visual processing, including increased baseline gamma oscillation power, increased theta/gamma phase amplitude coupling following stimulus presentation and lack of habituation to repetitive auditory stimuli. These alterations were also present in the human SYNGAP1-ID population as well, suggesting that they may be valid as reliable translational biomarkers of sensory processing alterations associated with SYNGAP1/Syngapl haploinsufficiency. In the Syngap1 $1^{+/-}$mouse model, we further found increased interictal activity during immobility, abnormal neural entrainment at different, sensory modality-specific frequencies and abnormal deviant sound detection. These phenomena could be explored in the human patient population as well to determine to what extent these alterations are conserved.

Interictal activity during immobility. One of the most frequent neurological problems in SYNGAP1-ID patients is the occurrence of epileptic encephalopathy. Extensive work showed the presence of epileptic phenotypes in different mouse models of Syngapl haploinsufficiency ${ }^{14,15,43}$. Classically, researchers have focused on seizure duration, and their frequency, however seizures may appear with a low frequency and their observation requires either long-term recording ${ }^{15}$ or the need to induce them using drugs or sensory stimulation ${ }^{14}$. Interictal spikes are pathological 
electrical events that reflect seizure susceptibility in patients and share common mechanisms with seizures $^{44}$. In the Syngap1 ${ }^{+/-}$mouse model, we found a relatively high frequency of interictal spikes, which was already evident in the first 15 minutes of recording for all Syngap1 ${ }^{+/-}$mice and in the different brain regions examined. This finding is consistent with what is previously reported in two different Syngapl haploinsufficient mice models as well as in SYNGAPl-ID patients ${ }^{14,}{ }^{15}$. Therefore, inter-ictal events, in particular during sleep and behavioral state transitions, could be a reliable and easy to measure biomarker for the evaluation of the efficacy of drug treatments on cortical hyper-excitability in SYNGAP1 associated disorders.

Translational biomarkers in baseline activity and sensory processing. Event Related Potentials (ERP) are frequently used as quantitative methods to directly compare sensory processing in patients with neurodevelopmental disorders and the corresponding mouse models ${ }^{16,}{ }^{17}$, nevertheless we did not find a consistent ERP phenotype either across sensory modalities or between species in the case of Syngap1/SYNGAP1 haploinsufficiency. A potential confounding effect is the use of different anti-epileptic medications in SYNGAPl-ID patients, which could affect ERP dynamics or amplitude. On the other hand, PAC analysis performed in the same data set revealed significant and consistently increased theta/gamma PAC following visual and auditory stimulation in Syngap1 ${ }^{+/}$mice and SYNGAP1-ID patients. Whether the augmented PAC is triggered by sensory stimulation in Syngap1 ${ }^{+/}$mice and SYNGAP1-ID patients or whether it reflects a basal disorganised network remains to be elucidated. Nevertheless, while PAC is an emerging tool mostly used to study local and long range connectivity ${ }^{64}$, recent studies support its potential interest as an EEG biomarker of different neurological pathologies ${ }^{41,65,66}$. For example, enhanced PAC has been extensively reported in epileptic patients and mouse models of epilepsy ${ }^{65}$, 67-69, thus leading to the hypothesis that increased PAC reflects network hyper-excitability ${ }^{70}$. Increased PAC has also been reported in other disorders such as obsessive-compulsive disorder ${ }^{71}$ and Parkinsons disease ${ }^{72}$. In particular, PAC levels appear proportional to the severity of motor symptoms in Parkinsons disease ${ }^{66,72}$, suggesting that excessive PAC "may reflect a pathological state in which the cortex is restricted to a monotonous pattern of coupling, rendering it less able to respond dynamically to signals from other cortical regions, such as frontal executive areas involved in internally directed movement"72. A similar rigid network organization could underlie the increased PAC observed in Syngap1/SYNGAP1 haploinsufficiency. 
Here, we observed a consistent increase in baseline gamma band power across all the regions we recorded in Syngapl mutant mice and SYNGAPl-ID patients. A recent study found alterations in the gamma power modulation during behavioral-state transitions in Syngap1 ${ }^{+/}$mice $^{15}$. Interestingly, this same study revealed a higher occurrence of myoclonic seizures during behavioral-state transitions in Syngap $1^{+/-}$mice and possibly SYNGAP1-ID patients ${ }^{15}$. Altogether, these observations highlight a potential link between the two phenomena, which could share common pathophysiological mechanisms. Indeed, several studies have shown that networks characterized by more prominent gamma oscillations are more likely to generate ictal activity, thus suggesting that increased gamma power may play a role in seizure generation ${ }^{73,74}$. For instance, in photosensitive epileptic patients, an increase in phase synchrony in the gamma-band $(30-120 \mathrm{~Hz})$ preceded an increased synchronization during flicker stimulation, but only when such stimulation lead to epileptic photoparoxysmal responses ${ }^{75}$. Of note, two recent studies showed that $<70 \%$ of awake seizures occurred at eye closure or with eyes closed in a cohort of SYNGAP1-ID patients with developmental epileptic encephalopathy ${ }^{76,77}$, supporting the hypothesis that cortical networks may be more prone to seizures even in absence of stimulation in SYNGAP1-ID.

Enhanced baseline gamma power has been described also in the auditory cortex of Fragile $\mathrm{X}$ syndrome and related mouse models (Fmrl KO mice $)^{25,58,78}$. Using an auditory chirp stimulation, the same studies showed that inter-trial phase synchrony was reduced in both humans with Fragile $\mathrm{X}$ syndrome and Fmrl KO mice, particularly at gamma frequency ${ }^{25}$, 58. These data lead to the hypothesis that enhanced background gamma oscillation, or network noise, may interfere with stimulus-evoked synchronization in Fragile X syndrome. In contrast, Syngap1 ${ }^{+-}$mice showed enhanced inter-trial phase synchrony when challenged with either a chirp or rhythmic auditory stimulation at $40 \mathrm{~Hz}$, but not $10 \mathrm{~Hz}$, suggesting that increased baseline gamma band power does not directly lead to deficits in mounting a specific gamma frequency-locked response to sounds. On the other hand, we observed significantly reduced entrainment in the visual cortex of Syngap1 $1^{+/-}$ mice specifically following a rhythmic sensory stimulation at $8 \mathrm{~Hz}$. These observations suggest that different neural circuit changes may underlie distinct neural entrainment alteration in visual versus auditory cortex. Neural entrainment may enhance signal-to-noise ratio in the local cortical network, thus increasing sensory gain for the specific frequency ${ }^{55}$. Thus, different region-specific neural entrainment alterations (enhanced in auditory cortex versus weakened in visual cortex) in 
Syngap $1^{+/-}$mice may indicate different effects on sensory processing and perception depending on the sensory modality.

In line with this hypothesis, we observed increased AEP amplitude responses to pure tones in auditory cortex, but reduced VEP amplitude responses to visual stimulation in Syngap1 ${ }^{+/-}$mice. In addition, a recent study showed hyposensitivity to touch in somatosensory cortex in another Syngap $1^{+/-}$mutant model ${ }^{22}$. These observations suggest that Syngapl haploinsufficiency does not lead to generalized hyper or hyposensitivity to sensory stimuli, but to cortical region-specific deficits, possibly relying on diverse distinct neuronal circuit alterations.

Potential contribution of GABAergic circuit deficits to sensory processing alterations. While the cellular bases underlying the generation of specific brain rhythms are not yet completely understood, extensive work point to a critical role of GABAergic interneurons, in particular parvalbumin-expressing (PV) cells, the larger subgroup of cortical GABAergic cells, which target the perisomatic domain of pyramidal neurons, and somatostatin (SST)-expressing, dendritic targeting interneurons ${ }^{79-81}$. SYNGAP1 protein is expressed by GABAergic cells and Syngap1 deletion in single PV cells in cortical organotypic cultures lead to reduced perisomatic synapse density $^{82}$. Several of the specific EEG alterations we observed in Syngap1 ${ }^{+/}$mice are consistent with PV cell circuit deficits. First, PV cells play a key role in generating and maintaining gamma oscillations in the brain ${ }^{83,84}$. In particular, recent studies have also shown that deficiency in PV interneuron-mediated inhibition contribute to increased baseline cortical gamma rhythm ${ }^{79,85,86}$, a phenotype we observed in both mutant mice and SYNGAP1-ID patients (Figure 2). Second, auditory habituation has been shown to involve an increase in inhibition ${ }^{87,88}$. Specifically, PV cells have been shown to amplify habituation providing non-specific inhibition. For instance, optogenetic suppression of PV cells led to increased responses to both repetitive (standard) and rare (deviant) tones ${ }^{87}$, which is similar to what we observed in Syngap ${ }^{+/}$-mice (Figure 7). Third, recent studies showed that optogenetically suppressing PV cell activity increased theta/gamma coupling in visual cortex ${ }^{79}$, suggesting that increased theta/gamma PAC (Figure 4) might indicate reduced PV cell activity. Further studies will be needed to clarify how and to what extent cortical PV cell circuit connectivity and function are altered by Syngapl haploinsufficiency and whether these alterations might be different in different cortical regions. 
Furthermore, we cannot exclude the involvement of different interneuron-specific dysfunctions in Syngapl haploinsufficiency. For example, SST interneurons selectively reduced excitatory responses to frequent tones in an oddball paradigm ${ }^{87}$ and optogenetic suppression of SOMs cells reduced the $\mathrm{MMN}$ peak ${ }^{89}$, suggesting impaired deviant sound detection, similarly to what we observed in Syngap1 ${ }^{+/-}$mice (Figure 7). Altogether these observations point to the contribution of GABAergic interneurons to the observed sensory perception phenotypes in Syngap1/SYNGAP1 haploinsufficiency. Further studies are needed to address the specific GABAergic network alterations and their relative contribution to different aspects of sensory processing and perception.

In conclusion, this study shows that visual and auditory perception is altered in Syngap1/SYNGAP1 haploinsufficiency, and reveal multiple novel robust and translation relevant phenotypes, which could both be used as biomarkers to monitor therapeutic outcomes of treatments and provide opportunities to better understand the pathophysiology of Syngap1/SYNGAP1 haploinsufficiency.

\section{ACKNOWLEDGEMENTS}

We thank Dr. Jean Marc Lina for his insightful suggestions on PAC analysis. We would like to thank James Bellord Waldron and Antônia Samia Fernandes do Nascimento for their technical assistance, the Comité Institutionnel de Bonne Pratiques Animales en Recherche (CIBPAR), all the personnel of the animal facility of the Research Center of CHU Sainte-Justine (Universite de Montreal) and the iPEDNeuroD human EEG platform (S.L.) for their instrumental technical support.

\section{FUNDINGS}

This work was supported by the Canadian Institutes of Health Research (M.A., J.M., G.DC.), Natural Sciences and Engineering Research Council of Canada (G.DC, S.L.), ERA-Net NEURON/DECODE! grant (G. DC), le Fond de Recherche du Québec en Santé (S.L.), Jonathan-Bouchard Chair in intellectual disability (J.M.) and Le Fondation des Étoiles (S.L., G.DC), M.I.C-M is supported by Overcôme Syngap1 Fondation.

\section{COMPETING INTERESTS}

The authors declare not competing interests. 


\section{REFERENCES}

1. Hamdan FF, Gauthier J, Araki Y, et al. Excess of de novo deleterious mutations in genes associated with glutamatergic systems in nonsyndromic intellectual disability. Am J Hum Genet. Mar 11 2011;88(3):306-16. doi:10.1016/j.ajhg.2011.02.001

2. Hamdan FF, Gauthier J, Spiegelman D, et al. Mutations in SYNGAP1 in autosomal nonsyndromic mental retardation. $N$ Engl $J$ Med. Feb 5 2009;360(6):599-605. doi:10.1056/NEJMoa0805392

3. Berryer MH, Chattopadhyaya B, Xing P, et al. Decrease of SYNGAP1 in GABAergic cells impairs inhibitory synapse connectivity, synaptic inhibition and cognitive function. Nat Commun. Nov 9 2016;7:13340. doi:10.1038/ncomms13340

4. Parker MJ, Fryer AE, Shears DJ, et al. De novo, heterozygous, loss-of-function mutations in SYNGAP1 cause a syndromic form of intellectual disability. American Journal of Medical Genetics Part A. 2015;167(10):2231-2237. doi:doi:10.1002/ajmg.a.37189

5. Mignot C, von Stülpnagel C, Nava C, et al. Genetic and neurodevelopmental spectrum of <em>SYNGAP1</em>-associated intellectual disability and epilepsy. Journal of Medical Genetics. 2016;53(8):511-522. doi:10.1136/jmedgenet-2015-103451

6. Agarwal M, Johnston MV, Stafstrom CE. SYNGAP1 mutations: Clinical, genetic, and pathophysiological features. Int $J$ Dev Neurosci. Nov 2019;78:65-76. doi:10.1016/j.ijdevneu.2019.08.003

7. Holder JL, Jr., Hamdan FF, Michaud JL. SYNGAP1-Related Intellectual Disability. In: Adam MP, Ardinger HH, Pagon RA, et al, eds. GeneReviews $((R)) .1993$.

8. Vlaskamp DRM, Shaw BJ, Burgess R, et al. SYNGAP1 encephalopathy: A distinctive generalized developmental and epileptic encephalopathy. Neurology. Jan 8 2019;92(2):e96-e107. doi:10.1212/WNL.0000000000006729

9. Deciphering Developmental Disorders S. Large-scale discovery of novel genetic causes of developmental disorders. Nature. Mar 12 2015;519(7542):223-8. doi:10.1038/nature14135

10. Deciphering Developmental Disorders S. Prevalence and architecture of de novo mutations in developmental disorders. Nature. Feb 23 2017;542(7642):433-438. doi:10.1038/nature21062

11. Gamache TR, Araki Y, Huganir RL. Twenty Years of SynGAP Research: From Synapses to Cognition. J Neurosci. Feb 19 2020;40(8):1596-1605. doi:10.1523/JNEUROSCI.0420-19.2020

12. Clement JP, Aceti M, Creson TK, et al. Pathogenic SYNGAP1 mutations impair cognitive development by disrupting maturation of dendritic spine synapses. Cell. Nov 9 2012;151(4):709723. doi:10.1016/j.cell.2012.08.045

13. Kilinc M, Creson T, Rojas C, et al. Species-conserved SYNGAP1 phenotypes associated with neurodevelopmental disorders. Mol Cell Neurosci. Sep 2018;91:140-150. doi:10.1016/j.men.2018.03.008 
14. Creson TK, Rojas C, Hwaun E, et al. Re-expression of SynGAP protein in adulthood improves translatable measures of brain function and behavior. Elife. Apr 26 2019;8doi:10.7554/eLife.46752

15. Sullivan BJ, Ammanuel S, Kipnis PA, Araki Y, Huganir RL, Kadam SD. Low-Dose Perampanel Rescues Cortical Gamma Dysregulation Associated With Parvalbumin Interneuron GluA2 Upregulation in Epileptic Syngap1(+/-) Mice. Biol Psychiatry. May 1 2020;87(9):829-842. doi:10.1016/j.biopsych.2019.12.025

16. Modi ME, Sahin M. Translational use of event-related potentials to assess circuit integrity in ASD. Nat Rev Neurol. Mar 2017;13(3):160-170. doi:10.1038/nrneurol.2017.15

17. LeBlanc JJ, DeGregorio G, Centofante E, et al. Visual evoked potentials detect cortical processing deficits in Rett syndrome. Ann Neurol. Nov 2015;78(5):775-86. doi:10.1002/ana.24513

18. Marco EJ, Hinkley LB, Hill SS, Nagarajan SS. Sensory processing in autism: a review of neurophysiologic findings. Pediatr Res. May 2011;69(5 Pt 2):48R-54R. doi:10.1203/PDR.0b013e3182130c54

19. Robertson CE, Baron-Cohen S. Sensory perception in autism. Nat Rev Neurosci. Nov 2017;18(11):671-684. doi:10.1038/nrn.2017.112

20. Siper PM, Zemon V, Gordon J, et al. Rapid and Objective Assessment of Neural Function in Autism Spectrum Disorder Using Transient Visual Evoked Potentials. PLoS One. 2016;11(10):e0164422. doi:10.1371/journal.pone.0164422

21. Javitt DC, Sweet RA. Auditory dysfunction in schizophrenia: integrating clinical and basic features. Nat Rev Neurosci. Sep 2015;16(9):535-50. doi:10.1038/nrn4002

22. Michaelson SD, Ozkan ED, Aceti M, et al. SYNGAP1 heterozygosity disrupts sensory processing by reducing touch-related activity within somatosensory cortex circuits. Nat Neurosci. Dec 2018;21(12):1-13. doi:10.1038/s41593-018-0268-0

23. Komiyama NH, Watabe AM, Carlisle HJ, et al. SynGAP regulates ERK/MAPK signaling, synaptic plasticity, and learning in the complex with postsynaptic density 95 and NMDA receptor. J Neurosci. Nov 15 2002;22(22):9721-32.

24. McLean AC, Valenzuela N, Fai S, Bennett SA. Performing vaginal lavage, crystal violet staining, and vaginal cytological evaluation for mouse estrous cycle staging identification. $J$ Vis Exp. Sep 15 2012;(67):e4389. doi:10.3791/4389

25. Lovelace JW, Ethell IM, Binder DK, Razak KA. Translation-relevant EEG phenotypes in a mouse model of Fragile X Syndrome. Neurobiol Dis. Jul 2018;115:39-48. doi:10.1016/j.nbd.2018.03.012

26. Cote V, Lalancette E, Knoth IS, et al. Distinct patterns of repetition suppression in Fragile $\mathrm{X}$ syndrome, down syndrome, tuberous sclerosis complex and mutations in SYNGAP1. Brain Res. Jan 15 2021;1751:147205. doi:10.1016/j.brainres.2020.147205

27. Tucker DM. Spatial sampling of head electrical fields: the geodesic sensor net. Electroencephalogr Clin Neurophysiol. Sep 1993;87(3):154-63.

28. Luck SJ. An introduction to the event-related potential technique. 2005:374. 
29. Saura S. Cognitive Auditory Evoked Potentials in Investigation of Hearing Discrimination. 2012 ;

30. Basirat A, Dehaene S, Dehaene-Lambertz G. A hierarchy of cortical responses to sequence violations in three-month-old infants. Cognition. Aug 2014;132(2):137-50. doi:10.1016/j.cognition.2014.03.013

31. Plank M. Downloads \& support ocular correction ICA. Brain. 2013;49(9):1-4.

32. Oostenveld R, Fries P, Maris E, Schoffelen JM. FieldTrip: Open source software for advanced analysis of MEG, EEG, and invasive electrophysiological data. Comput Intell Neurosci. 2011;2011:156869. doi:10.1155/2011/156869

33. Herrmann CS, Grigutsch M, Busch NA. EEG oscillations and wavelet analysis. Eventrelated potentials: A methods handbook. MIT Press; 2005:229-259:chap 11.

34. Makeig S, Debener S, Onton J, Delorme A. Mining event-related brain dynamics. Trends in Cognitive Sciences. 2004/05/01/ 2004;8(5):204-210. doi:https://doi.org/10.1016/j.tics.2004.03.008

35. Tallon-Baudry C, Bertrand O. Oscillatory gamma activity in humans and its role in object representation. Trends in Cognitive Sciences. 4/1/ 1999;3(4):151-162. doi:http://dx.doi.org/10.1016/S1364-6613(99)01299-1

36. Delorme A, Makeig S. EEGLAB: an open source toolbox for analysis of single-trial EEG dynamics including independent component analysis. Journal of Neuroscience Methods. 2004/03/15/ 2004;134(1):9-21. doi:https://doi.org/10.1016/j.jneumeth.2003.10.009

37. Lachaux J-P, Rodriguez E, Martinerie J, Varela FJ. Measuring phase synchrony in brain signals. Human Brain Mapping. 1999;8(4):194-208. doi:doi:10.1002/(SICI)10970193(1999)8:4<194::AID-HBM4>3.0.CO;2-C

38. Aru J, Aru J, Priesemann V, et al. Untangling cross-frequency coupling in neuroscience. Curr Opin Neurobiol. Apr 2015;31:51-61. doi:10.1016/j.conb.2014.08.002

39. Dvorak D, Fenton AA. Toward a proper estimation of phase-amplitude coupling in neural oscillations. J Neurosci Methods. Mar 30 2014;225:42-56. doi:10.1016/j.jneumeth.2014.01.002

40. Seymour RA, Rippon G, Kessler K. The Detection of Phase Amplitude Coupling during Sensory Processing. Front Neurosci. 2017;11:487. doi:10.3389/fnins.2017.00487

41. Seymour RA, Rippon G, Gooding-Williams G, Schoffelen JM, Kessler K. Dysregulated oscillatory connectivity in the visual system in autism spectrum disorder. Brain. Oct 1 2019;142(10):3294-3305. doi:10.1093/brain/awz214

42. Hamdan FF, Daoud H, Piton A, et al. De novo SYNGAP1 mutations in nonsyndromic intellectual disability and autism. Biol Psychiatry. May 1 2011;69(9):898-901. doi:10.1016/j.biopsych.2010.11.015

43. Ozkan ED, Creson TK, Kramar EA, et al. Reduced cognition in Syngap1 mutants is caused by isolated damage within developing forebrain excitatory neurons. Neuron. Jun 18 2014;82(6):1317-33. doi:10.1016/j.neuron.2014.05.015 
44. Karoly PJ, Freestone DR, Boston R, et al. Interictal spikes and epileptic seizures: their relationship and underlying rhythmicity. Brain. Apr 2016;139(Pt 4):1066-78. doi:10.1093/brain/aww019

45. Cardin JA. Snapshots of the Brain in Action: Local Circuit Operations through the Lens of gamma Oscillations. $J \quad$ Neurosci. Oct $12 \quad$ 2016;36(41):10496-10504. doi:10.1523/JNEUROSCI.1021-16.2016

46. Gabard-Durnam LJ, Wilkinson C, Kapur K, Tager-Flusberg H, Levin AR, Nelson CA. Longitudinal EEG power in the first postnatal year differentiates autism outcomes. Nat Commun. Sep 13 2019;10(1):4188. doi:10.1038/s41467-019-12202-9

47. Levin AR, Varcin KJ, O'Leary HM, Tager-Flusberg H, Nelson CA. EEG power at 3 months in infants at high familial risk for autism. J Neurodev Disord. Sep 13 2017;9(1):34. doi:10.1186/s11689-017-9214-9

48. Peiker I, David N, Schneider TR, Nolte G, Schottle D, Engel AK. Perceptual Integration Deficits in Autism Spectrum Disorders Are Associated with Reduced Interhemispheric GammaBand Coherence. J Neurosci. Dec 16 2015;35(50):16352-61. doi:10.1523/JNEUROSCI.144215.2015

49. Milne E, Scope A, Pascalis O, Buckley D, Makeig S. Independent component analysis reveals atypical electroencephalographic activity during visual perception in individuals with autism. Biol Psychiatry. Jan 1 2009;65(1):22-30. doi:10.1016/j.biopsych.2008.07.017

50. Grent-'t-Jong T, Gross J, Goense J, et al. Resting-state gamma-band power alterations in schizophrenia reveal E/I-balance abnormalities across illness-stages. Elife. Sep 27 2018;7doi:10.7554/eLife.37799

51. McNally JM, McCarley RW. Gamma band oscillations: a key to understanding schizophrenia symptoms and neural circuit abnormalities. Curr Opin Psychiatry. May 2016;29(3):202-10. doi:10.1097/YCO.0000000000000244

52. Spencer KM, Niznikiewicz MA, Shenton ME, McCarley RW. Sensory-evoked gamma oscillations in chronic schizophrenia. Biol Psychiatry. Apr 15 2008;63(8):744-7. doi:10.1016/j.biopsych.2007.10.017

53. Knoth IS, Vannasing P, Major P, Michaud JL, Lippé S. Alterations of visual and auditory evoked potentials in fragile X syndrome. International Journal of Developmental Neuroscience. 2014/08/01/ 2014;36:90-97. doi:https://doi.org/10.1016/j.ijdevneu.2014.05.003

54. Gandal MJ, Edgar JC, Ehrlichman RS, Mehta M, Roberts TP, Siegel SJ. Validating gamma oscillations and delayed auditory responses as translational biomarkers of autism. Biol Psychiatry. Dec 15 2010;68(12):1100-6. doi:10.1016/j.biopsych.2010.09.031

55. Obleser J, Kayser C. Neural Entrainment and Attentional Selection in the Listening Brain. Trends Cogn Sci. Oct 9 2019;doi:10.1016/j.tics.2019.08.004

56. Wilson TW, Rojas DC, Reite ML, Teale PD, Rogers SJ. Children and adolescents with autism exhibit reduced MEG steady-state gamma responses. Biol Psychiatry. Aug 1 2007;62(3):192-7. doi:10.1016/j.biopsych.2006.07.002 
57. Ethridge LE, White SP, Mosconi MW, Wang J, Byerly MJ, Sweeney JA. Reduced habituation of auditory evoked potentials indicate cortical hyper-excitability in Fragile $\mathrm{X}$ Syndrome. Transl Psychiatry. Apr 19 2016;6:e787. doi:10.1038/tp.2016.48

58. Ethridge LE, White SP, Mosconi MW, et al. Neural synchronization deficits linked to cortical hyper-excitability and auditory hypersensitivity in fragile X syndrome. Mol Autism. 2017;8:22. doi:10.1186/s13229-017-0140-1

59. Seymour RA, Rippon G, Gooding-Williams G, Sowman PF, Kessler K. Reduced auditory steady state responses in autism spectrum disorder. Mol Autism. Jul 1 2020;11(1):56. doi:10.1186/s13229-020-00357-y

60. Paul A, Nawalpuri B, Shah D, Sateesh S, Muddashetty RS, Clement JP. Differential Regulation of Syngap1 Translation by FMRP Modulates eEF2 Mediated Response on NMDAR Activity. Front Mol Neurosci. 2019;12:97. doi:10.3389/fnmol.2019.00097

61. Sinclair D, Oranje B, Razak KA, Siegel SJ, Schmid S. Sensory processing in autism spectrum disorders and Fragile X syndrome-From the clinic to animal models. Neuroscience \& Biobehavioral Reviews. 2017/05/01/ 2017;76:235-253. doi:http://dx.doi.org/10.1016/j.neubiorev.2016.05.029

62. Garrido MI, Kilner JM, Stephan KE, Friston KJ. The mismatch negativity: a review of underlying mechanisms. Clin Neurophysiol. Mar 2009;120(3):453-63. doi:10.1016/j.clinph.2008.11.029

63. Naatanen R, Sussman ES, Salisbury D, Shafer VL. Mismatch negativity (MMN) as an index of cognitive dysfunction. Brain Topogr. Jul 2014;27(4):451-66. doi:10.1007/s10548-014-0374-6

64. Canolty RT, Edwards E, Dalal SS, et al. High gamma power is phase-locked to theta oscillations in human neocortex. Science. Sep 15 2006;313(5793):1626-8. doi:10.1126/science. 1128115

65. Samiee S, Levesque M, Avoli M, Baillet S. Phase-amplitude coupling and epileptogenesis in an animal model of mesial temporal lobe epilepsy. Neurobiol Dis. Jun 2018;114:111-119. doi:10.1016/j.nbd.2018.02.008

66. Devergnas A, Caiola M, Pittard D, Wichmann T. Cortical Phase-Amplitude Coupling in a Progressive Model of Parkinsonism in Nonhuman Primates. Cereb Cortex. Jan 1 2019;29(1):167177. doi:10.1093/cercor/bhx314

67. Maheshwari A, Akbar A, Wang M, et al. Persistent aberrant cortical phase-amplitude coupling following seizure treatment in absence epilepsy models. $J$ Physiol. Dec 1 2017;595(23):7249-7260. doi:10.1113/JP274696

68. Zhang R, Ren Y, Liu C, et al. Temporal-spatial characteristics of phase-amplitude coupling in electrocorticogram for human temporal lobe epilepsy. Clin Neurophysiol. Sep 2017;128(9):1707-1718. doi:10.1016/j.clinph.2017.05.020

69. Alvarado-Rojas C, Valderrama M, Fouad-Ahmed A, et al. Slow modulations of highfrequency activity $(40-140-\mathrm{Hz})$ discriminate preictal changes in human focal epilepsy. Sci Rep. Apr 1 2014;4:4545. doi:10.1038/srep04545

70. Canolty RT, Knight RT. The functional role of cross-frequency coupling. Trends Cogn Sci. Nov 2010;14(11):506-15. doi:10.1016/j.tics.2010.09.001 
71. Bahramisharif A, Mazaheri A, Levar N, Richard Schuurman P, Figee M, Denys D. Deep Brain Stimulation Diminishes Cross-Frequency Coupling in Obsessive-Compulsive Disorder. Biol Psychiatry. Oct 1 2016;80(7):e57-8. doi:10.1016/j.biopsych.2015.05.021

72. de Hemptinne C, Ryapolova-Webb ES, Air EL, et al. Exaggerated phase-amplitude coupling in the primary motor cortex in Parkinson disease. Proc Natl Acad Sci U S A. Mar 19 2013;110(12):4780-5. doi:10.1073/pnas.1214546110

73. Hermes D, Kasteleijn-Nolst Trenite DGA, Winawer J. Gamma oscillations and photosensitive epilepsy. Curr Biol. May 8 2017;27(9):R336-R338. doi:10.1016/j.cub.2017.03.076

74. Perry G, Brindley LM, Muthukumaraswamy SD, Singh KD, Hamandi K. Evidence for increased visual gamma responses in photosensitive epilepsy. Epilepsy Res. Aug 2014;108(6):1076-86. doi:10.1016/j.eplepsyres.2014.04.012

75. Parra J, Kalitzin SN, Iriarte J, Blanes W, Velis DN, Lopes da Silva FH. Gamma-band phase clustering and photosensitivity: is there an underlying mechanism common to photosensitive epilepsy and visual perception? Brain. May 2003;126(Pt 5):1164-72. doi:10.1093/brain/awg109

76. von Stulpnagel C, Funke C, Haberl C, et al. SYNGAP1 Mutation in Focal and Generalized Epilepsy: A Literature Overview and A Case Report with Special Aspects of the EEG. Neuropediatrics. Aug 2015;46(4):287-91. doi:10.1055/s-0035-1554098

77. Lo Barco T, Kaminska A, Solazzi R, et al. SYNGAP1-DEE: A visual sensitive epilepsy. Clin Neurophysiol. Apr 2021;132(4):841-850. doi:10.1016/j.clinph.2021.01.014

78. Wang J, Ethridge LE, Mosconi MW, et al. A resting EEG study of neocortical hyperexcitability and altered functional connectivity in fragile X syndrome. J Neurodev Disord. 2017;9:11. doi:10.1186/s11689-017-9191-z

79. Chen G, Zhang Y, Li X, et al. Distinct Inhibitory Circuits Orchestrate Cortical beta and gamma Band Oscillations. Neuron. Dec 20 2017;96(6):1403-1418 e6. doi:10.1016/j.neuron.2017.11.033

80. Buzsaki G, Wang XJ. Mechanisms of gamma oscillations. Annu Rev Neurosci. 2012;35:203-25. doi:10.1146/annurev-neuro-062111-150444

81. Amilhon B, Huh CY, Manseau F, et al. Parvalbumin Interneurons of Hippocampus Tune Population Activity at Theta Frequency. Neuron. Jun 3 2015;86(5):1277-89. doi:10.1016/j.neuron.2015.05.027

82. Berryer MH, Chattopadhyaya B, Xing P, et al. Decrease of SYNGAP1 in GABAergic cells impairs inhibitory synapse connectivity, synaptic inhibition and cognitive function. Article. Nature Communications. 11/09/online 2016;7:13340. doi:10.1038/ncomms 13340

https://www.nature.com/articles/ncomms13340\#supplementary-information

83. Csicsvari J, Jamieson B, Wise KD, Buzsaki G. Mechanisms of gamma oscillations in the hippocampus of the behaving rat. Neuron. Jan 23 2003;37(2):311-22. doi:10.1016/s08966273(02)01169-8

84. Cardin JA, Carlen M, Meletis K, et al. Driving fast-spiking cells induces gamma rhythm and controls sensory responses. Nature. Jun 4 2009;459(7247):663-7. doi:10.1038/nature08002 
85. Guyon N, Zacharias LR, Oliveira EFd, et al. Network asynchrony underlying increased broadband gamma power. bioRxive. 2020;

86. Carlen M, Meletis K, Siegle JH, et al. A critical role for NMDA receptors in parvalbumin interneurons for gamma rhythm induction and behavior. Mol Psychiatry. May 2012;17(5):537-48. doi:10.1038/mp.2011.31

87. Natan RG, Briguglio JJ, Mwilambwe-Tshilobo L, et al. Complementary control of sensory adaptation by two types of cortical interneurons. Elife. Oct 13 2015;4doi:10.7554/eLife.09868

88. Kato HK, Gillet SN, Isaacson JS. Flexible Sensory Representations in Auditory Cortex Driven by Behavioral Relevance. Neuron. Dec 2 2015;88(5):1027-1039. doi:10.1016/j.neuron.2015.10.024

89. Hamm JP, Yuste R. Somatostatin Interneurons Control a Key Component of Mismatch Negativity in Mouse Visual Cortex. Cell Rep. Jul 19 2016;16(3):597-604. doi:10.1016/j.celrep.2016.06.037 